\title{
Understanding the Fundamentals of Motor Current Signature Analysis
}

\author{
M.D. Shirley ${ }^{1}$,A.R. Champneys ${ }^{2} \dagger$, N. MArrin $^{2}$, M.R. Moore ${ }^{1}$, B.M.A.G \\ PietTe $^{3}$, and A.D. ShaW ${ }^{4}$ \\ ${ }^{1}$ University of Oxford, Oxford, UK \\ ${ }^{2}$ University of Bristol, Bristol, UK \\ ${ }^{3}$ University of Durham, Durham, UK \\ ${ }^{4}$ Swansea University, Swansea, UK
}

(Communicated to MIIR on 28 January 2022)

Study Group: ESGI 162. Leeds (virtual), UK, 20 - 24 July 2020

Communicated by: J.A. Ward

Industrial Partner: Faraday Predictive ltd

Presenter: Geoff Walker, Andy Bates

Team Members: A. Bates, Faraday Predictive; A.R. Champneys, Univ. Bristol; J.M.C. Correia, Univ. Évora; Y. Farah, Univ. Birmingham; G. Gajjar, IIT Bombay; M.J. Hunt, Univ. Warwick; A.A. Lacey, Heriot-Watt Univ.; N. Marrin, Univ. Bristol; K. Mora, Paderborn Univ.; M.R. Moore, Univ. Oxford H. Ockendon, Univ. Oxford; J.R. Ockendon, Univ. Oxford; D. Patel, Maharaja Sayajirao Univ; B.M.A.G Piette, Univ. Durham; James Roscoe, Univ. Liverpool; A.D. Shaw, Swansea Univ.; M.D. Shirley, Univ. Oxford; G. Walker, Faraday Predictive

Industrial Sector: Electronics

Tools: ODEs, Classical mechanics, Asymptotics, Numerical methods

Key Words: Induction motor, Residual current

MSC2020 Codes: 70,94,78

$\dagger$ Corresponding Author: A.R.Champneys@bristol.ac.uk 


\section{Summary}

Motor current signature analysis is a method to perform on-line monitoring of the condition of electrical motors by examining the spectrum of the residual current of the motor for sidebands around the main frequencies. The interpretation of these spectra would be improved by having a physics based understanding of how particular perturbations appear in a spectrum. This report considers two possible models for an induction motor and its residual current.

The first model considered uses the Steinmetz equivalent circuit, a standard model from the literature. The second model is derived from first principles based on Faraday and Ohms law and the conservation of angular momentum. It is able to capture the coupling between the rotor dynamics and the residual current.

Some initial results are given for each model using both asymptotic and numerical techniques. They indicate that both models can produce sidebands, although the physical model captures the complicated asymmetric behaviour seen in real spectrums. The modelling in this report is a good basis that with further analysis should be able to explain why particular sidebands are observed when particular defects are present.

\section{Challenge}

Faraday Predictive monitor the behaviour of equipment driven by electric motor, by measuring the voltage and current drawn by the motor, and by analysing the change in relationship between voltage and current. Effectively we are using the distortions on the current waveform that have not been caused by distortions on the voltage waveform as an indication of what is going on in the combined (motor and driven equipment) system - as that is the only place that can have caused the distortions. This approach has been used pragmatically for years, but not all aspects of it are fully understood.

At the ESGI in Cambridge in 2019, we presented a problem involving some unusual signal patterns we had been seeing in the distortion spectrum. That work made significant progress on aspects of both nonlinear signal processing and the forward problem of the nonlinear dynamics of rotors with rub and contact. It has already spawned master's projects in two Universities $[14,18,19]$ and enhanced engagements with some of key customers.

At this year's ESGI we want to look at the physics that underlies our technology at a more fundamental level. In particular, we would like to understand better the relative impact of changes in torque on the motor shaft versus changes in eccentricity of the rotor in the motor, both of which are known to affect the current. We would also like to compare the magnitude of these with changes in the magnetic behaviour of the rotor, which is the classic application of classic Motor Current Signature Analysis - used to detect deterioration of the structure of the motor rotor.

We would like to understand the relative magnitude and likely spectral shape effects of these two types of phenomena - ones that cause the rotor to run eccentrically, and 
ones that cause changes in the torque on the rotor - and how their magnitude compares with those from cracked rotors.

\subsubsection{Problem 1 - What causes our fundamental signal?}

From the basic equations for electric current and voltage in a three-phase induction motor, can you derive from first principles the effect on stator current of a) a small change in load (torque) on the motor, and b) of a small change in eccentricity of the rotor within the stator. How does the magnitude of signals from torsional or radial movement compare with signals from rotor magnetic asymmetry (ie cracked rotor bars)? Also, to what extent does motor speed need to change, or is it just a change in acceleration of the rotor, to create a change in stator current? How does the answer change for a permanent magnet (or other form of synchronous) motor?

\subsubsection{Problem 2. What determines how and (where) signals appear on the spectrum?}

We classically expect signals to appear as side bands on a carrier frequency, so that for example a $10 \mathrm{~Hz}$ signal with a $50 \mathrm{~Hz}$ supply would appear as two peaks, at $10 \mathrm{~Hz}$ either side of the main $50 \mathrm{~Hz}$ peak, ie at $40 \mathrm{~Hz}$ and $60 \mathrm{~Hz}$ - as per the diagram in Figure 1. Strictly, the signal can appear at $n f_{\text {line }} \pm m f_{p}$ where $n$ and $m$ are integers, i.e. we can have multiple sidebands, either on each side of the fundamental, or on higher harmonics of the fundamental. So the specific questions to answer are: What causes signal to appear as multiple sidebands on the same carrier frequency? What causes signals to appear as sidebands on fundamental and higher harmonics of the carrier frequency? (and what determines which harmonics it will appear on?) What causes signals to appear as sidebands more strongly on one side than the other of the carrier frequency? (and what determines if it is the lower or higher frequency side?)

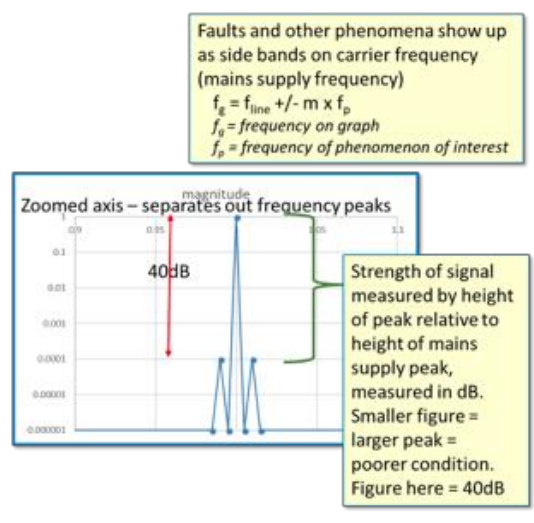

Figure 1. Example spectrum. 


\section{Introduction and problem formulation}

In order to address both the problems presented by Faraday we will focus on analysing and developing models for three-phase induction motors. As depicted in Figure 2 a three phase induction motor consists of a stator made up of electro-magnets. An alternating current power supply is applied to the stator, creating a magnetic field that rotates at the frequency of the applied current. This magnetic field induces a counter current in the rotor, a series of closed conducting loops. This current creates force due to Faraday's law on each side of the wire, causing it to begin rotating. A shaft is attached to the rotor and is used to drive external machinery.

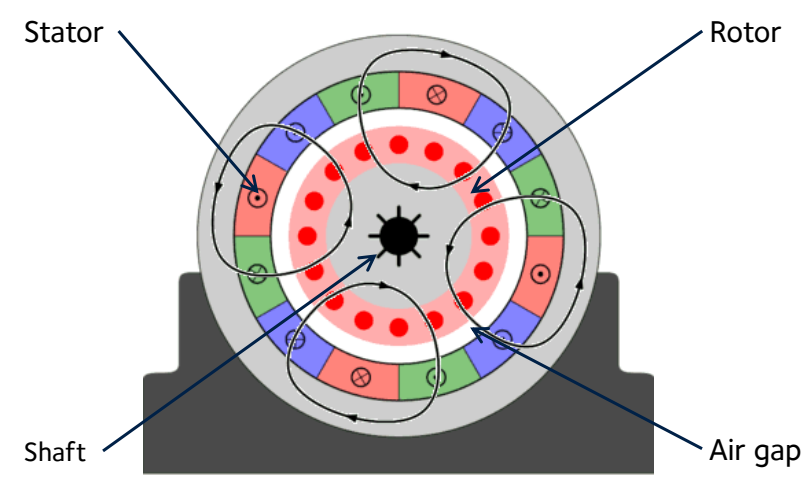

Figure 2. A three-phase induction motor. From [4].

By measuring the voltage applied to the motor and the current of the motor, a residual current, the current that cannot be explained by applied voltage, is found. Examining the spectrum of the residual current reveals additional peaks caused by physical processes affecting the motor. Typical faults in the motor can be: damage to loops in the rotor, eccentricity of the rotor, and variations in the applied load.

\section{Literature review}

At the study group a literature review was undertaken to ascertain what previous work had been done using a detailed physics based model to explain and interpret motor current signature analysis results. An early, key paper in the field by Cameron, Thomson and Dow (1986) [8] which describes methods for calculating the magnetic permeability in the stator based on harmonic distortions in the air gap. We will now outline the key developments identified within the over 600 papers citing this article:

- Thomson and Fenger (2001) [27] gives examples of the signatures observed in the spectra of residual currents, obtained from measurements of motors whose faults include: broken rotor bars, shorted turns in low-voltage stator windings, and abnormal airgap eccentricity.

- Nandi et al (2002) [23] simulate the effect of both static and dynamic eccentricity on a 
residual currents spectrum. They show that both high- and low-frequency components must be measured under different load conditions to identify eccentricity related faults.

- Nandi et al (2001) [22] describes how the observed harmonics typically depend on the number of rotor slots (which can vary widely) and on the number of pole pairs (usually within one to four), however note that not all three phase induction motors are capable of generating such harmonics.

- Nandi (2004) [21] contains some mathematical expressions for the effect on the magnetic field and torque of various slot anomalies in the motor.

- Cusido et al (2006) [10] reports that Motor Current Signature Analysis (MCSA) method presents some problems when the load torque is not constant with the rotation angle shaft: harmonics related to the non constant load overlap with the fundamental harmonics and difficulties appear applied to medium and high power engines working at low speed (frequency separation) or low load torque (small slip). Authors propose the injection of an additional voltage at frequency different from the fundamental and then to study the resulting harmonics.

- Ghoggal et al (2009) [13] consider air gaps and slot skewing in the motor and how to calculate in theory the effect on the current spectrum based on these faults. This is compared with experiment.

- Pennacci (2009) [24] provides a simulation of the electrical generator of a large industrial machine and identifies sources of nonlinearity in the equations, which lead to limit cycles in phase space plots. They also identify what this means in terms of the spectrum.

- Faiz et al (2010) [11] contains some theory and some nice spectral plots explaining how different eccentricity faults contribute to different parts of the observed spectrum.

- Rezazadeh et al (2011) [20] and Zhang et al (2010) [28] contain a systematic review of motor current signature analysis and other techniques for vibration detection in induction motors, up to 2011. A more recent review is provided by Salah (2019) [25] which includes expressions for the forces caused by motor eccentricities, and the corresponding magnetic fields and harmonic signatures you might expect to see.

- Aggarwal et al (2019) [6] is a recent paper that appears to use the Cameron et al theory, provide more detailed mathematical explanation, and to apply this as a practical method.

- Bouras et al (2020) [7] are mainly concerned with the detection and diagnosis of bearing faults for which they use MCSA (Motors Current Signature Analysis) supplemented with Park Vector Approach Orbital (OPVA) and Gabor Spectrogram (STFT), say 'spectral, orbital and temporal signatures', whose simultaneous application helps to provide the signature separation of near defects overlapping in time and/or frequency.

- Leite et al (2015) [17] discusses the use of filtering frequency bands to detect faults.

\section{Equivalent circuit model}

In this section, we analyse a standard model of induction motors, the Steinmetz equivalent circuit, using numerical and asymptotic techniques. We examine how periodic perturbations to the slip (as defined in (4.1)) can cause side-bands in the stator current spectrum and give a formula to determine the slip based on stator current measurements. 


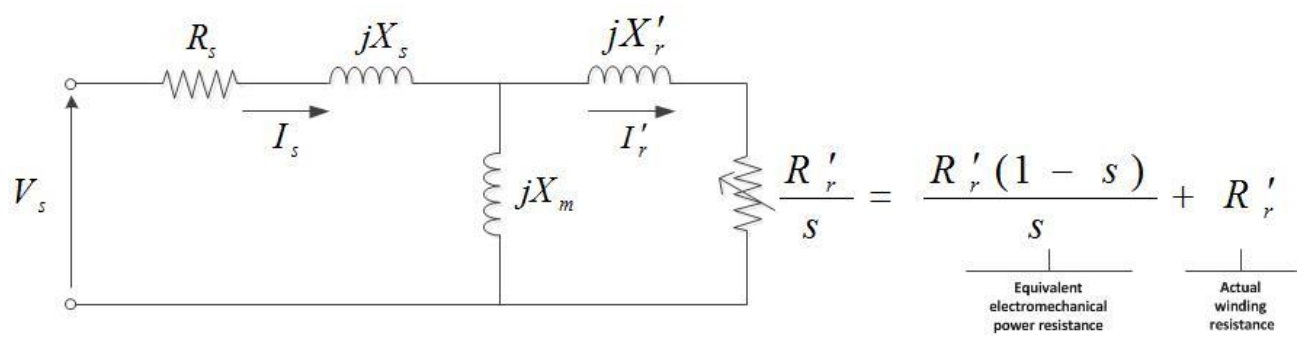

Figure 3. The Steinmetz equivalent circuit. From [4].

\subsection{The Steinmetz equivalent circuit}

In electrical engineering, equivalent circuit models are a common tool for modelling electrical devices. We shall focus on the Steinmetz equivalent circuit model, depicted in Figure 3, as it is the IEEE recommended equivalent circuit for an induction motor [4]. It is a single phase represention of a multiphase induction motor. Here $V_{s}, R_{s}, X_{s}$ are the stator potential difference, resistance, and reactance, $R_{r}^{\prime}, X_{r}$ are the rotor resistance and leakage reactance, and $I_{s}, I_{r}^{\prime}$, and $I_{m}$ are the stator current, rotor current, and inductor current. The slip $s$ is defined as

$$
s=\frac{\text { stator electrical speed }- \text { rotor mechanical speed }}{\text { stator electrical speed }},
$$

and gives the relative difference between the stator speed and rotor speed. For a motor with no load $0<s \ll 1$, and for a locked motor $0<1-s \ll 1$.

Using Kirchhoff's circuit laws [2], we can transform the Steinmetz equivalent circuit into the following system of ordinary differential equations:

$$
\begin{aligned}
I_{s} & =I_{r}^{\prime}-I_{m}, \\
R_{s} I_{s}+L_{s} \dot{I}_{s}-L_{m} \dot{I}_{m} & =V_{s}, \\
L_{r}^{\prime} \dot{I}_{r}^{\prime}+\frac{R_{r}^{\prime}}{s} I_{r}^{\prime}+L_{m} \dot{I}_{m} & =0 .
\end{aligned}
$$

In (4.2)-(4.4), $L_{s}, L_{m}$, and $L_{r}$ are the inductances corresponding to the stator, inductor, and rotor, which can be calculated from the reactances by

$$
X=2 \pi f L,
$$

where $f$ is the frequency of the signal (in our case the applied potential difference). As we are modelling an alternating current induction motor the applied potential difference will oscillate, hence we take it to be of the form

$$
V=\sqrt{2} V_{\text {rms }} \cos (2 \pi f t),
$$

where $V_{\text {rms }}$ is the root mean squared magnitude of applied potential difference.

Typical values for the Steinmetz model of a induction motor, taken from [16] are given in Table 1. 
Table 1. Typical dimensional parameter values taken from [16].

$\begin{array}{cc}\text { Parameter } & \text { Value } \\ f & 50 \mathrm{~Hz} \\ R_{s} & 2.3 \Omega \\ R_{r}^{\prime} & 4.2 \Omega \\ X_{s} & 17.5 \times 10^{-3} \Omega \\ X_{r}^{\prime} & 17.5 \times 10^{-3} \Omega \\ X_{m} & 0.23 \Omega \\ V_{\mathrm{rms}} & 90 \mathrm{~V} \\ s & 0.05\end{array}$

\subsection{Nondimensionalisation}

We nondimensionalise variables by introducing the scalings

$$
\left(I_{s}, I_{r}^{\prime}, I_{m}\right)=\frac{V_{\mathrm{rms}}}{R_{r}^{\prime}}\left(\hat{I}_{s}, \hat{I}_{r}^{\prime}, \hat{I}_{m}\right), V=V_{\mathrm{rms}} \hat{V}, \text { and } t=\frac{1}{2 \pi f} \hat{t},
$$

so that (4.2)-(4.4) become

$$
\begin{aligned}
\hat{I}_{s} & =\hat{I}_{r}^{\prime}-\hat{I}_{m}, \\
R_{\mathrm{rat}} I_{s}+(X / R)_{s, r} \dot{\hat{I}}_{s}-(X / R)_{m, r} \dot{\hat{I}}_{m} & =\hat{V}_{s}, \\
(X / R)_{r, r} s \dot{\hat{I}}_{r}^{\prime}+I_{r}^{\prime}+(X / R)_{m, r} s \dot{I}_{m} & =0
\end{aligned}
$$

where

$$
\hat{V}_{s}=\sqrt{2} \cos (\hat{t}),
$$

and

$$
R_{\mathrm{rat}}=\frac{R_{s}}{R_{r}^{\prime}}, \quad(X / R)_{m, r}=\frac{X_{m}}{R_{r}^{\prime}}, \quad(X / R)_{r, r}=\frac{X_{r}^{\prime}}{R_{r}^{\prime}}, \quad(X / R)_{s, r}=\frac{X_{s}}{R_{r}^{\prime}}
$$

are nondimensional parameters. The timescale has been chosen so that the dominant frequency of the system is one. For the remainder of this section we drop the hat notation for brevity.

\subsection{Constant slip}

We first restrict ourselves to the case of constant slip. This models a motor with no mechanical defect. Then we can reduce (4.8)-(4.10) to a single ODE for $I_{s}$,

$$
A s \ddot{I}_{s}+\left(B R_{\mathrm{rat}} s+C\right) \dot{I}_{s}+R_{\mathrm{rat}} I_{s}=B s \dot{V}+V .
$$

where

$$
\begin{array}{r}
A=(X / R)_{m, r}(X / R)_{r, r}+(X / R)_{m, r}(X / R)_{s, r}+(X / R)_{r, r}(X / R)_{s, r}, \\
B=(X / R)_{m, r}+(X / R)_{r, r}, C=(X / R)_{m, r}+(X / R)_{s, r} .
\end{array}
$$

The details of the derivation of this ODE is given in Appendix A.

We can solve (4.13) using the numerical scheme outlined in Appendix A. The current and spectrum resulting from doing this with the parameter values listed in Table 1 is shown in Figure 4b. For initial conditions we take $I_{s}=0$ and $\dot{I}_{s}=0$. To avoid any 
interference from the choice of initial data we shall calculate the spectrum using data from a late time. Specifically, we shall extract the spectrum from the solution from $t=1$ to $t=1+100 / f$ to calculate the spectrum. In Figure $4 \mathrm{~b}$, we note that the only peak on the spectrum is at $50 \mathrm{~Hz}$, no sidebands appear or harmonics appear.

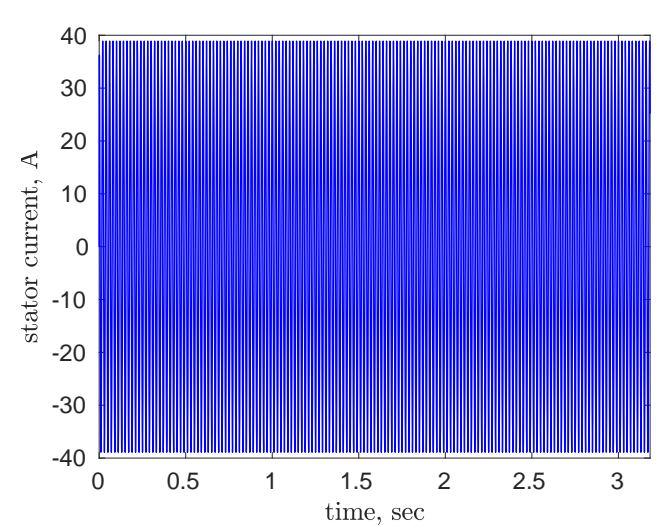

(a) Stator current with constant slip.

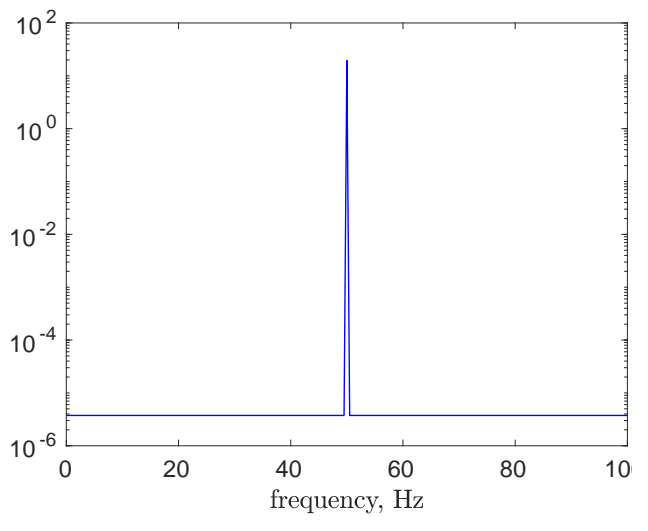

(b) Stator current spectrum with constant slip.

Figure 4. Numerical solution of constant slip Steinmetz model given by (4.13)-(4.14) with parameter values from Table 1.

\subsection{Variable slip}

In real motors variations in the load will cause the slip to vary. We therefore consider how small periodic perturbations to slip will appear in the stator current's spectrum. If we assume that the slip is a function of time then (4.8)-(4.10) again can be reduced to a single equation

$$
A s \ddot{I}_{s}+\left[A \dot{s}+B R_{\mathrm{rat}} s+C\right] \dot{I}_{s}+\left[B R_{\mathrm{rat}} \dot{s}+R_{\mathrm{rat}}\right] I_{s}=B s \dot{V}+B \dot{s} V+V .
$$

where $A, B$, and $C$ are as defined in (4.14). We shall restrict our attention to sinusoidal perturbations of slip of the form

$$
s(t)=s_{0}+\epsilon \cos (2 \pi \phi t),
$$

where $\phi$ is the frequency of the perturbation and $0<\epsilon \ll 1$. We denote the dimensionless frequency of the perturbation by $\hat{\phi}=\phi / f$. At this point we note that with the parameter values given in Table 1, both (4.13) and (4.15) are overdamped second order ODEs. This should be the case for any realistic parameter values, as to avoid a motor's speed exceeding its safety threshold, electric motors are designed to be overdamped. Hence, transitions between desired rotor speeds, or recovery from perturbations, are achieved monotonically, rather than in a potentially dangerous oscillatory manner, as would be the case for weak damping.

We can solve (4.15) numerically using the scheme outlined in Appendix A, using the parameter values in Table 1 and $\epsilon=0.005, \phi=1 \mathrm{~Hz}$. The initial conditions are $I_{s}=$ 
$0, \dot{I}_{s}=0$. The resulting current and spectrum are plotted in Figure 5. Even though (4.15) is heavily overdamped, we see the existence of symmetrical sidebands in the solution spectrum at $49 \mathrm{~Hz}$ and $51 \mathrm{~Hz}$.

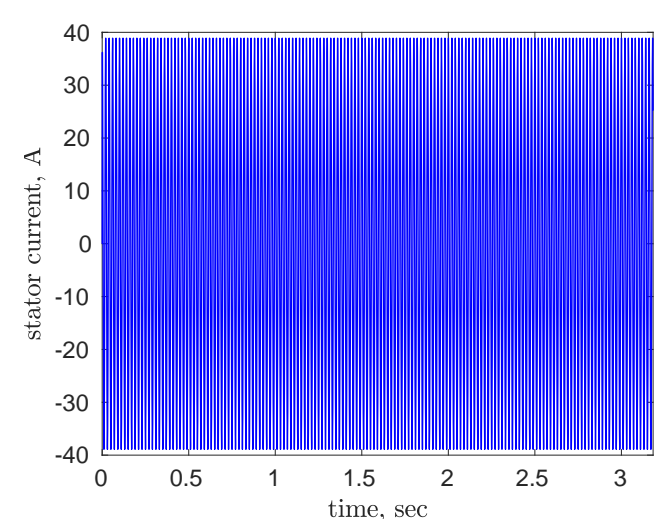

(a) Stator current with varying slip.

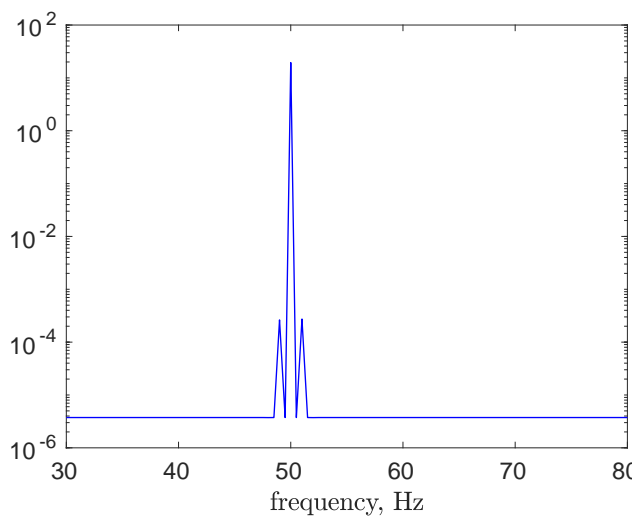

(b) Stator current spectrum with constant slip.

Figure 5. Numerical solution of the variable slip Steinmetz model with parameter values from Table 1.

\subsection{Asymptotic results}

We shall try to understand the existence of these sidebands by performing an asymptotic analysis of (4.15)-(4.16) in the limit that $\epsilon \rightarrow 0$.

To investigate this, we consider also consider this situation asymptotically. To simplify the problem we first rescale $s$ and $t$ to simplify the problem. Let

$$
s=\frac{C}{B R_{\mathrm{rat}}} \tilde{s}, \text { and } t=\frac{A}{B R_{\mathrm{rat}}} \tilde{t},
$$

then (4.15) becomes

$$
\ddot{I}_{s}+\left(\frac{\dot{\tilde{s}}+1}{\tilde{s}}+1\right) \dot{I}_{s}+\left(\frac{\dot{\tilde{s}}+\Gamma}{\tilde{s}}\right) I_{s}=\frac{1}{R_{\mathrm{rat}}}\left(\dot{V}+\frac{\dot{\tilde{s}}}{\tilde{s}} V+\frac{\Gamma}{\tilde{s}} V\right),
$$

where

$$
\Gamma=\frac{A}{B C}, \Omega=\frac{B R_{\mathrm{rat}}}{A}, \psi=\phi \frac{B R_{\mathrm{rat}}}{A}, V(\tilde{t})=\sqrt{2} \cos (\Omega \tilde{t}), s(\tilde{t})=s_{0}+\cos (\psi \tilde{t})
$$

We now drop the tilde notation for simplicity.

If we now expand $I_{s}=I_{0}+\epsilon I_{1}+O\left(\epsilon^{2}\right)$, we have at leading order

$$
\ddot{I}_{0}+\left(\frac{1}{s_{0}}+1\right) \dot{I}_{0}+\frac{\Gamma}{s_{0}} I_{0}=\frac{\sqrt{2}}{R_{\text {rat }}}\left(-\Omega \sin (\Omega t)+\frac{\Gamma}{s_{0}} \cos (\Omega t)\right),
$$

If we assume that we are in the realistic situation where the system is overdamped, the solution to the homogeneous problem (with right-hand side set to zero) is

$$
I_{0}=a \exp \left(\left|\lambda_{+}\right| t\right)+b \exp \left(\left|\lambda_{-}\right| t\right)
$$


where $a, b$ are arbitary constants determined by the inital conditions and

$$
\lambda_{ \pm}=\frac{1}{2}\left(-\frac{1}{s_{0}}-1 \pm \sqrt{\left(\frac{1}{s_{0}}+1\right)^{2}-\frac{4 \Gamma}{s_{0}}}\right) .
$$

Henceforth, we shall pursue the analysis in the steady state limit in which motion due to the initial conditions has died away. Thus, we shall concentrate on the particular integral

$$
I_{0}=c \cos ((\Omega t)+d \cos ((\Omega t),
$$

where it is straightforward to show that $c$ and $d$ satisfy

$$
\left(\begin{array}{cc}
\frac{\Gamma}{s_{0}}-\Omega^{2} & \Omega\left(\frac{1}{s_{0}}+1\right) \\
-\Omega\left(\frac{1}{s_{0}}+1\right) & \frac{\Gamma}{s_{0}}-\Omega^{2}
\end{array}\right)\left(\begin{array}{l}
c \\
d
\end{array}\right)=\left(\begin{array}{c}
\frac{\sqrt{2} \Gamma}{R_{\mathrm{rat}} s_{0}} \\
-\frac{\sqrt{2} \Omega}{R_{\mathrm{rat}}}
\end{array}\right) .
$$

At next order, we compare terms of $O(\epsilon)$, and we see that

$$
\begin{aligned}
\ddot{I}_{1}+\left(\frac{1}{s_{0}}+1\right) \dot{I}_{1}+\frac{\Gamma}{s_{0}} I_{1}= & \left(\frac{1}{s_{0}^{2}} \cos (\psi t)+\frac{\psi}{s_{0}} \sin (\psi t)\right) \dot{I}_{0}+\left(\frac{\psi}{s_{0}} \sin (\psi t)+\frac{\Gamma}{s_{0}^{2}} \cos (\psi t)\right) I_{0} \\
& -\frac{\sqrt{2}}{R_{\mathrm{rat}}}\left(\frac{\psi}{s_{0}} \cos (\Omega t) \sin (\psi t)+\frac{\Gamma}{s_{0}^{2}} \cos (\Omega t) \cos (\psi t)\right) \\
= & \left(\frac{1}{s_{0}^{2}} \cos (\psi t)+\frac{\psi}{s_{0}} \sin (\psi t)\right)(-c \Omega \sin (\Omega t)+d \Omega \cos (\Omega t)) \\
& +\left(\frac{\psi}{s_{0}} \sin (\psi t)+\frac{\Gamma}{s_{0}^{2}} \cos (\psi t)\right)(+c \cos (\Omega t)+d \sin (\Omega t)) \\
& -\frac{\sqrt{2}}{R_{\mathrm{rat}}}\left(\frac{\psi}{s_{0}} \cos (\Omega t) \sin (\psi t)+\frac{\Gamma}{s_{0}^{2}} \cos (\Omega t) \cos (\psi t)\right)
\end{aligned}
$$


Upon grouping terms on the right-hand side, we see that

$$
\begin{aligned}
\ddot{I}_{1}+\left(\frac{1}{s_{0}}+1\right) \dot{I}_{1}+\frac{\Gamma}{s_{0}} I_{1}= & \left(-\frac{\Gamma \sqrt{2}}{s_{0}^{2} R_{\text {rat }}}+\frac{\Omega c}{s_{0}^{2}}+\frac{\Gamma d}{s_{0}^{2}}\right) \cos (\psi t) \cos (\Omega t) \\
& +\left(-\frac{\Omega d}{s_{0}^{2}}+\frac{\Gamma c}{s_{0}^{2}}\right) \cos (\psi t) \sin (\Omega t) \\
& +\left(-\frac{\psi \sqrt{2}}{s_{0} R_{\text {rat }}}+\frac{\psi \Omega c}{s_{0}}+\frac{\psi d}{s_{0}}\right) \sin (\psi t) \cos (\Omega t) \\
& +\left(-\frac{\psi \Omega d}{s_{0}}+\frac{\psi c}{s_{0}}\right) \sin (\psi t) \sin (\Omega t) \\
= & \frac{1}{2}\left(-\frac{\Gamma \sqrt{2}}{s_{0}^{2} R_{\text {rat }}}+\frac{\Omega c}{s_{0}^{2}}+\frac{\Gamma d}{s_{0}^{2}}+\frac{\psi \Omega d}{s_{0}}-\frac{\psi c}{s_{0}}\right) \cos ((\Omega+\psi) t) \\
& +\frac{1}{2}\left(-\frac{\Gamma \sqrt{2}}{s_{0}^{2} R_{r a t}}+\frac{\Omega c}{s_{0}^{2}}+\frac{\Gamma d}{s_{0}^{2}}-\frac{\psi \Omega d}{s_{0}}+\frac{\psi c}{s_{0}}\right) \cos ((\Omega-\psi) t) \\
& +\frac{1}{2}\left(-\frac{\psi \sqrt{2}}{s_{0} R_{r a t}}+\frac{\psi \Omega c}{s_{0}}+\frac{\psi d}{s_{0}}-\frac{\Omega d}{s_{0}^{2}}+\frac{\Gamma c}{s_{0}^{2}}\right) \sin ((\Omega+\psi) t) \\
& +\frac{1}{2}\left(\frac{\psi \sqrt{2}}{s_{0} R_{\text {rat }}}-\frac{\psi \Omega c}{s_{0}}-\frac{\psi d}{s_{0}}-\frac{\Omega d}{s_{0}^{2}}+\frac{\Gamma c}{s_{0}^{2}}\right) \sin ((\Omega-\psi) t)
\end{aligned}
$$

where in the second inequality we have used standard trigonometric identities to reform the terms.

Therefore, clearly we must seek a particular solution of the form

$$
I_{1}^{*}=\alpha \cos ((\Omega+\psi) t)+\beta \cos ((\Omega-\psi) t)+\gamma \sin ((\Omega+\psi) t)+\delta \sin ((\Omega-\psi) t),
$$

we find

$$
\begin{gathered}
\left(\begin{array}{cc}
\frac{\Gamma}{s_{0}}-(\Omega+\psi)^{2} & \left(\frac{1}{s_{0}}+1\right)(\Omega+\psi) \\
-\left(\frac{1}{s_{0}}+1\right)(\Omega+\psi) & \frac{\Gamma}{s_{0}}-(\Omega+\psi)^{2}
\end{array}\right)\left(\begin{array}{l}
\alpha \\
\gamma
\end{array}\right)=\frac{1}{2}\left(\begin{array}{c}
-\frac{\Gamma \sqrt{2}}{s_{0}^{2} R_{\text {rat }}}+\frac{\Omega c}{s_{0}^{2}}+\frac{\Gamma d}{s_{0}^{2}}+\frac{\psi \Omega d}{s_{0}}-\frac{\psi c}{s_{0}} \\
-\frac{\psi \sqrt{2}}{s_{0} R_{r a t}}+\frac{\psi \Omega c}{s_{0}}+\frac{\psi d}{s_{0}}-\frac{\Omega d}{s_{0}^{2}}+\frac{\Gamma c}{s_{0}^{2}}
\end{array}\right) \\
\left(\begin{array}{cc}
\frac{\Gamma}{s_{0}}-(\Omega-\psi)^{2} & \left(\frac{1}{s_{0}}+1\right)(\Omega-\psi) \\
-\left(\frac{1}{s_{0}}+1\right)(\Omega-\psi) & \frac{\Gamma}{s_{0}}-(\Omega-\psi)^{2}
\end{array}\right)\left(\begin{array}{l}
\beta \\
\delta
\end{array}\right)=\frac{1}{2}\left(\begin{array}{c}
-\frac{\Gamma \sqrt{2}}{s_{0}^{2} R_{r a t}}+\frac{\Omega c}{s_{0}^{2}}+\frac{\Gamma d}{s_{0}^{2}}-\frac{\psi \Omega d}{s_{0}}+\frac{\psi c}{s_{0}} \\
\frac{\psi \sqrt{2}}{s_{0} R_{r a t}}-\frac{\psi \Omega c}{s_{0}}-\frac{\psi d}{s_{0}}-\frac{\Omega d}{s_{0}^{2}}+\frac{\Gamma c}{s_{0}^{2}}
\end{array}\right) .
\end{gathered}
$$

Thus, in this first-order analysis, we expect to see, in addition to a peak at $\Omega$, sidebands at $\Omega \pm \psi$. In particular, if we take a sinusoidal perturbation at $1 \mathrm{~Hz}$ on a motor running at $50 \mathrm{~Hz}$, these results suggest sidebands will appear at $49 \mathrm{~Hz}$ and $51 \mathrm{~Hz}$, which is precisely what occurs in Figure 5. We could determine the amplitudes for a particular problem by calculating the coefficients $c, d, \alpha, \beta, \gamma$ and $\delta$. Naturally, proceeding to higher order will show that further bands appear at other linear combinations of $\Omega$ and $\psi$. 


\subsection{Inverse problem for slip}

In some scenarios, it may be unrealistic to assume that the slip is known a priori, while we may well have an accurate idea of the stator current. If the stator current is known, then (4.15) can instead be viewed as a model for how the slip varies over time. Regrouping terms we have that

$$
\left(A \dot{I}_{s}+B R_{\mathrm{rat}} I_{s}+B V\right) \dot{s}+\left(A \ddot{I}_{s}+B R_{\mathrm{rat}} \dot{I}_{s}+B \dot{V}\right) s=V-C \dot{I}-R I,
$$

which we can integrate to find

$$
s(t)=\left(A \dot{I}_{s}+B R_{\mathrm{rat}} I_{s}+B V\right)^{-1} \int_{0}^{t} V(\tau)-C \dot{I}(\tau)-R I(\tau) \mathrm{d} \tau .
$$

\section{Physical model of an induction motor}

We now consider a second model for an induction motor, this time derived from first principles based on Faraday's law, Ohms law and conservation of angular momentum. The motivation for deriving this model is that the rotor dynamics will appear directly in the model, allowing a wider range of defects to be modelled directly.

Note that the notation between sections 4 , and sections 5-6 should be regarded as independent.

\subsection{Derivation of rotor model}

\subsubsection{Single loop}

We begin by considering a single closed, planar conducting loop of wire of length $l$, width $2 r$, and area $A=2 l r$, that is always tangent in the $x$ direction, as depicted in Figure 6, inside a magnetic field $\vec{B}(t)$, at time $t$. Then the loop has vector area

$$
\vec{S}(t)=A \vec{n}=A(0,-\sin \theta(t), \cos \theta(t)),
$$

where $\vec{n}(t)$ the unit vector normal to the loop, and $\theta(t)$ is the angle the planar normal of the loop the makes with the normal of the plane given by $z=0$. By assuming that the magnetic field is radially rotating with frequency $\Omega$, (i.e. the the frequency of the current applied to the stator), and constant strength $B$, we may write $\vec{B}$ as

$$
\vec{B}=B(0,-\sin (\Omega t), \cos (\Omega t))^{\mathrm{T}} .
$$
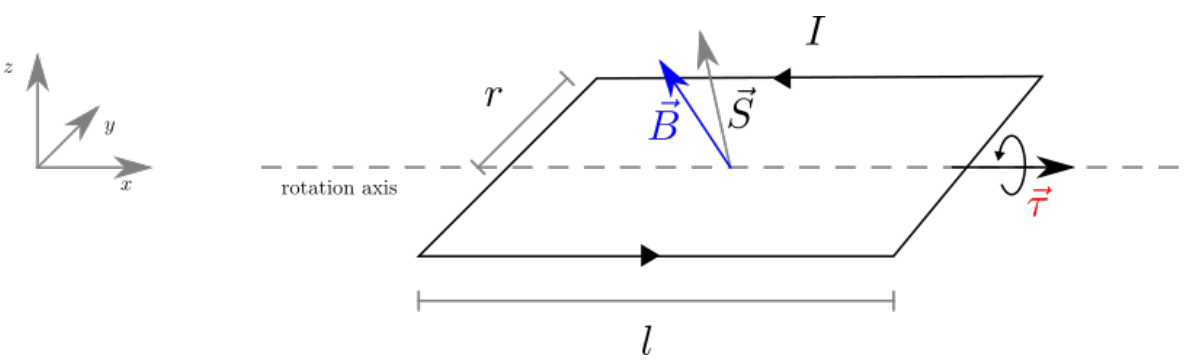

Figure 6. Single loop inside the rotor. 
There will be a magnetic flux $\Phi=\vec{B} \cdot \vec{S}$, through the loop which by Faraday's law of induction[1], induces an electromotive force $\mathcal{E}$, as it changes,

$$
\mathcal{E}=-\frac{\mathrm{d} \Phi}{\mathrm{d} t}=-\frac{\mathrm{d}(\vec{B} \cdot \vec{S})}{\mathrm{d} t} .
$$

As the loop is closed, we have that a current

$$
I=\frac{\mathcal{E}}{R}=-\frac{1}{R} \frac{\mathrm{d}(\vec{B} \cdot \vec{S})}{\mathrm{d} t},
$$

where $R$ is the resistance of the loop is induced.

This current then induces itself a Lorenz force $F$, given by

$$
\vec{F}=I \vec{l} \times \vec{B}
$$

where $\vec{l}$ is a vector whose magnitude is the length of wire, and whose direction is along the wire, aligned with the direction of flow of (conventional) current $I$ [5]. To find the torque $\tau$ about the $x$ axis, we only need to consider the angular component $I(\vec{I} \times \vec{B}) \cdot \vec{\theta}$. where $\vec{\theta}$ is an angular unit vector equal to $\pm \mathbf{n}$ for each side of the loop respectively. Since the sign of the current will be the same as the sign of $\vec{\theta}$ both sides will contribute to the total torque, giving

$$
\begin{aligned}
\tau & =-2 r \vec{n} \cdot(I \vec{l} \times \vec{B}), \\
& =2 r l I \vec{n} \cdot(\vec{B} \times \vec{x}), \\
& =A I \vec{n} \cdot(\vec{B} \times \vec{x}),
\end{aligned}
$$

where $x$ is the unit vector in the positive $x$ direction. Note that $\vec{B} \times \vec{n}$ will always be in the direction of $\vec{x}$, so we can write this compactly as

$$
\vec{\tau}=I(\vec{S} \times \vec{B}) .
$$

Combining (5.4) and (5.9) we find

$$
\tau=-\frac{\vec{S} \times \vec{B}}{R} \frac{\mathrm{d}(\vec{B} \cdot \vec{S})}{\mathrm{d} t} .
$$

Combining (5.1), (5.10), and (5.2) we can derive an expression for torque in terms of $\theta$

$$
\begin{aligned}
\vec{\tau} & =\frac{A B}{R} \sin (\theta-\Omega t) \frac{\mathrm{d}}{\mathrm{d} t}(A B \cos (\theta-\Omega t)) \vec{x}, \\
& =-\frac{A^{2} B^{2}}{R}(\dot{\theta}-\Omega) \sin ^{2}(\theta-\Omega t) \vec{x} .
\end{aligned}
$$

\subsubsection{Multiple loops}

Having described in detail the derivation of the torque experienced by a single closed wire loop in a magnetic field, we now consider modelling a rotor as $N$ closed wire loops 
rotating around the same axis and with an angle of $\pi / N$ between adjacent loops, as depicted in Figure 7. Letting $\phi_{i}=(i-1) \pi / N$ for $i=1,2, \ldots, N$, we can write the position of the ith loop as $\theta(t)+\phi_{i}$.

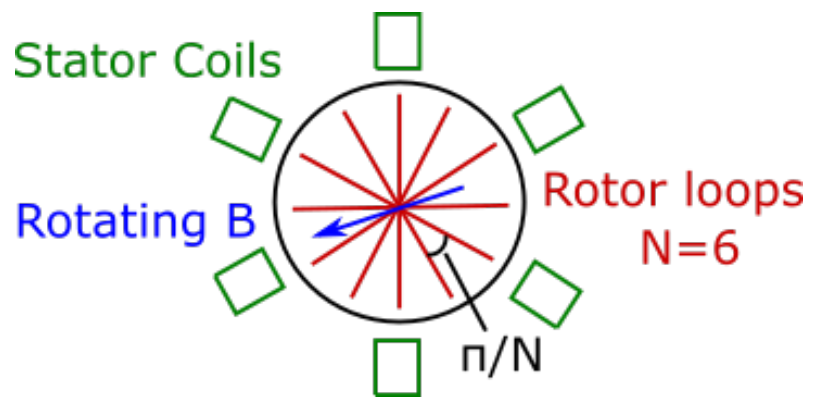

Figure 7. Setup of motor model.

We can now write down the an expression for the torque about the $x$ axis of the ith loop, $\vec{\tau}_{i}$. As we wish to consider the effect of time dependent defects, we generalise (5.12), to that for a time dependent $B$ and/or $A$,

$$
\vec{\tau}_{i}=\frac{\Phi_{i} \dot{\Phi}_{i}}{2 R} \sin \left(2\left(\theta-\Omega t+\phi_{i}\right)\right) \vec{x}-\frac{\Phi_{i}^{2}}{R}(\dot{\theta}-\Omega) \sin ^{2}\left(\theta-\Omega t+\phi_{i}\right) \vec{x},
$$

where $\Phi_{i}(t)=A_{i}(t) B_{i}(t)$.

\subsubsection{Conservation of angular momentum}

We can now consider the balance of moments of inertia about the $x$ axis, given by

$$
\begin{aligned}
\mathcal{I} \ddot{\theta} & =\sum_{i=1}^{N}\left(\vec{\tau}_{i}\right)_{x}+\mathcal{L}, \\
& =\sum_{i=1}^{N} \frac{\Phi_{i} \dot{\Phi}_{i}}{2 R} \sin \left(2\left(\theta-\Omega t+\phi_{i}\right)\right) \vec{x}-(\dot{\theta}-\Omega) \sum_{i=1}^{N} \frac{\Phi_{i}^{2}}{R} \sin ^{2}\left(\theta-\Omega t+\phi_{i}\right) \vec{x}+\mathcal{L}
\end{aligned}
$$

where $\mathcal{I}$ is inertia and $\mathcal{L}$ is the load.

The simplest, linear model for friction is

$$
\mathcal{L}=-\Gamma \dot{\theta},
$$

but a non-linear model

$$
\mathcal{L}=-\Gamma \dot{\theta}+\Gamma_{3} \dot{\theta}^{3},
$$

could also be used. We use a cubic drag, rather than the more common quadratic form $\Gamma_{2} \dot{\theta}|\dot{\theta}|$, for air resistance, so that the frictional force is a smooth nonlinear function, which simplifies the numerical solution.

If we consider the special case where all the loops are identical, so that $\Phi_{i}(t)=\Phi$, a constant, then (5.15) reduces to

$$
\mathcal{I} \ddot{\theta}=-\frac{N}{2} \frac{\Phi^{2}}{R}(\dot{\theta}-\Omega)+\mathcal{L} .
$$




\subsection{Reaction current in the stator}

Given a model for the rotor's angular position $\theta$ at time $t$, we now consider how to calculate the residual current measured in motor current signature analysis.

By (5.4), the current that has been induced in the rotor by loop $i$ is given by

$$
I_{i}=\frac{1}{R}\left(\Phi_{i}(\dot{\theta}-\Omega) \sin \left(\theta-\Omega t+\phi_{i}\right)-\dot{\Phi}_{i} \cos \left(\theta-\Omega t+\phi_{i}\right)\right) .
$$

This current will generate a magnetic field $\tilde{\vec{B}}_{i}$ inside the loop with an intensity proportional to the current. For a simple loop the lines of the magnetic field form closed loops around the conductor loop itself. For simplicity, we assume that this magnetic field is normal to the loop and given by

$$
\tilde{B}_{i}=k_{i} I_{i}=\frac{k_{i}}{R}\left(\Phi_{i}(\dot{\theta}-\Omega) \sin \left(\theta-\Omega t+\phi_{i}\right)-\dot{\Phi}_{i} \cos \left(\theta-\Omega t+\phi_{i}\right)\right),
$$

where $k_{i}$ is an unknown constant dependant on the geometry of the motor. As a vector field normal to the surface of the loop $\tilde{B}_{i}$ induces a potential difference, the counter electromotive force $\Psi_{i}$, upon the stator coils,

$$
V_{s}=-\frac{\mathrm{d} \Psi_{i}}{\mathrm{~d} t}=\frac{\mathrm{d}(\tilde{B} \cdot \tilde{S})}{\mathrm{d} t}=-A_{s} \frac{\mathrm{d}}{\mathrm{d} t}\left(\cos \left(\theta+\phi_{i}-\phi_{s}\right) \tilde{B}_{i}\right),
$$

where $A_{s}$ is the effective area of the stator coil (an unknown parameter) and $\phi_{s}$ is the angular position of the considered stator coil.

The stator coil forms an RL circuit, but the $\mathrm{R}$ is expected to be very small small, so we can approximate it as a simple $\mathrm{L}$ circuit. Then the current $I_{s, i}$ induced by the counter electromotive force satisfies [3]

$$
L_{s} \frac{\mathrm{d} I_{s}}{\mathrm{~d} t}=V_{s}=-A_{s} \frac{\mathrm{d}}{\mathrm{d} t}\left(\cos \left(\theta+\phi_{i}-\phi_{s}\right) \tilde{B}_{i}\right)
$$

by (5.21), where $L_{s}$ is the inductance of the stator coil. Hence, integrating and using $(5.20)$, we find

$$
\begin{aligned}
I_{s, i} & =-\frac{A_{s}}{L_{s}} \cos \left(\theta+\phi_{i}-\phi_{s}\right) \tilde{B}_{i} \\
& =-\frac{A_{s}}{L_{s}} \cos \left(\theta+\phi_{i}-\phi_{s}\right) \frac{k_{i}}{R}\left(\Phi_{i}(\dot{\theta}-\Omega) \sin \left(\theta-\Omega t+\phi_{i}\right)-\dot{\Phi}_{i} \cos \left(\theta-\Omega t+\phi_{i}\right)\right) .
\end{aligned}
$$

Taking all the rotor loops into account the total current in the stator $I_{s}$, will be

$$
I_{s}=-\frac{A_{s}}{L_{s}} \sum_{i=1}^{N} \cos \left(\theta+\phi_{i}-\phi_{s}\right) \frac{k_{i}}{R}\left(\Phi_{i}(\dot{\theta}-\Omega) \sin \left(\theta-\Omega t+\phi_{i}\right)-\dot{\Phi}_{i} \cos \left(\theta-\Omega t+\phi_{i}\right)\right) .
$$

If we assume all the loops to be identical, so that $\Phi_{i}=\Phi$ and $k_{i}=k$ for all $i$ then this can be reduced to

$$
I_{s}=\frac{N}{2} \frac{A_{s} k}{L_{s} R}\left((\dot{\theta}-\Omega) \Phi \sin \left(\Omega t-\phi_{s}\right)+\dot{\Phi} \cos \left(\Omega t-\phi_{s}\right)\right) .
$$


Note this result used the trigonometric identities $\sum_{i=1}^{N} \cos \left(\Theta+2 \phi_{i}\right)=0$ and $\sum_{i=1}^{N} \sin (\Theta+$ $\left.2 \phi_{i}\right)=0$ for all $\Theta$ and $N>1$.

The residual current, the current analysed by Faraday Predictive to predict faults, is the difference between the predicted current when the motor spins at constant frequency $\dot{\theta}=\Omega$, and the measured current. In our model the residual current given by the difference between (5.25) when $\theta=\Omega t$ and (5.25) for general $\theta$,

$$
I_{\text {res }}=\left.I_{s}\right|_{\dot{\theta}=\Omega}-I_{s}=\frac{A_{s}}{L_{s}} \sum_{i=1}^{N} \cos \left(\theta+\phi_{i}-\phi_{s}\right) \frac{k_{i}}{R} \Phi_{i}(\dot{\theta}-\Omega) \sin \left(\theta-\Omega t+\phi_{i}\right) .
$$

For a rotor with identical loops we obtain the simpler expression,

$$
I_{\text {res }}=\frac{N}{2} \frac{A_{s}}{L} \frac{k}{R}(\Omega-\dot{\theta}) \Phi \sin \left(\Omega t-\phi_{s}\right) .
$$

At the study group taking the continuum limit of (5.25), by letting $N \rightarrow \infty$ was considered. However, it is not clear that this is correct as the finite loops model was derived by assuming each loop could be treated independently, which would not be true if the loops are sufficiently close that their electromagnetic fields interact. For more details on continuum limits in electrical devices see [9] and [15] for discussion of the correct model of a Faraday cage.

A naive calculation can proceed as follows. If we let the number of rotor coils $N$ go to infinity, and we assume that the rotor is homogeneous, we have

$$
\begin{aligned}
I_{s}=-\frac{A_{s}}{L_{s}} \int_{0}^{\pi} & {\left[\cos \left(\theta+\phi-\phi_{s}\right) \frac{k(\phi)}{R}\right.} \\
& (\Phi(\phi)(\dot{\theta}-\Omega) \sin (\theta-\Omega t+\phi)-\dot{\Phi}(\phi) \cos (\theta-\Omega t+\phi))] \mathrm{d} \phi,
\end{aligned}
$$

where $k(\phi), \Phi(\phi, t)$ are the continuum limits of $k_{i}, \Phi_{i}$, giving their values at angular position $\phi$. If we assume that $k$ and $\Phi$ are constant with respect to $\phi$ (symmetric rotor) then (5.29) simplifies to

$$
I_{s}=\frac{\pi}{2} \frac{A_{s}}{L_{s}} \frac{k}{R}\left(\Phi(\dot{\theta}-\Omega) \sin \left(\Omega t-\phi_{s}\right)+\dot{\Phi} \cos \left(\Omega t-\phi_{s}\right)\right) .
$$

In this case the expression for the residual current $I_{\text {res }}$ reduces to

$$
I_{\text {res }}=\frac{\pi}{2} \frac{A_{s}}{L_{s}} \frac{k}{R} \Phi(\Omega-\dot{\theta}) \sin \left(\Omega t-\phi_{s}\right)
$$

\subsubsection{Spectrum of residual current}

To detect defects in a motor the spectrum of the residual current is analysed. To do this theoretically using our model we must first solve (5.15) to give $\theta$, using a particular load model. Then (5.27) can be solve to give $I_{r e s}$ and the absolute value of its Fourier transform can be computed.

Many of the parameters in the problem are not fully known, but they will only affect the amplitude of the spectra, and to a lesser extent the phase of the spectra. We therefore chose to set $R=1, \Phi_{i}=\Phi=1$ for all $i$, and $A_{s} k_{i} / 2 L_{s} R=0.001$ in their respective units. Taking $\Phi_{i}$ equal for all $i$ models a motor without defect. We have also chosen 
$\Omega=50 \mathrm{~Hz}$ and set the number of rotor loops to $N=16$. We restrict our load model to the linear one, taking $\Gamma_{3}=0$, and choose $\Gamma=0.2$ so that the slip frequency $\Omega-\dot{\theta}$ is approximately $1.22 \mathrm{~Hz}$.

For the initial condition for (5.15) we take $\theta(0)=0$ and $\dot{\theta}(0)=47.5 \mathrm{~Hz}$. To get periodic data to calculate the Fourier transform we let the system relax for $10 \mathrm{sec}$ so that the motor reaches its cruising speed and integrate the equation for another 300 second, sampling the data at $1 \mathrm{kHz}$.

The ODE (5.15) is sovled using the 4th order Runge-Kutta method and the Fourier transform was computed using the an FFT with $2^{18}$ data points multiplied by a Hamming window. We then computed the absolute value of the Fourier transform of the residual current given by (5.27).

We have carried out this calculation for a motor without defect, and plotted the resulting residual current spectrum in Figure 8 and present it on a logarithmic plot. We would expect the spectrum to be a delta function with its spike at $\Omega=50 \mathrm{~Hz}$, but the numerical evaluation on a finite domain leads to the spread seen spectrum in the figure.

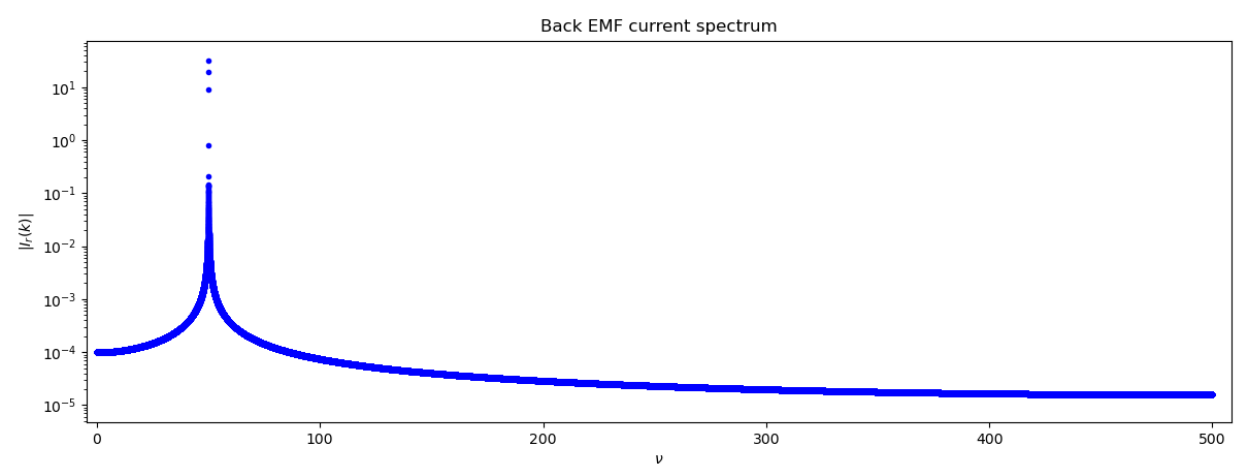

Figure 8. Spectrum of a motor without defect.The spread of the spectrum is due to a non perfect sampling. Slip frequency: $1.22 \mathrm{~Hz}$.

\subsection{Modelling defects}

We will now demonstrate how our model can be used model a range of defects quite easily.

\subsubsection{Single damaged bar in the rotor}

We begin by assuming that one of the loops, say loop $j$, of the rotor has a defect. Then the value of $\Phi_{j}$ will differ from that of $\Phi_{i}=\Phi$ for $i \neq j$, and we assume it to be $\Phi_{j}=\Phi(1+\epsilon)$ where, to be consistent with our previous parameter choice in section 5.2.1, $\Phi=1$. This models a difference in flux through rotor loop $j$. Taking $\epsilon=0.1$ we obtain the spectrum shown on figure 9 .

We see that in this case, the spectrum exhibits 2 asymmetric spikes around the main's frequency and the frequency difference is twice the slip frequency. We also see a third 


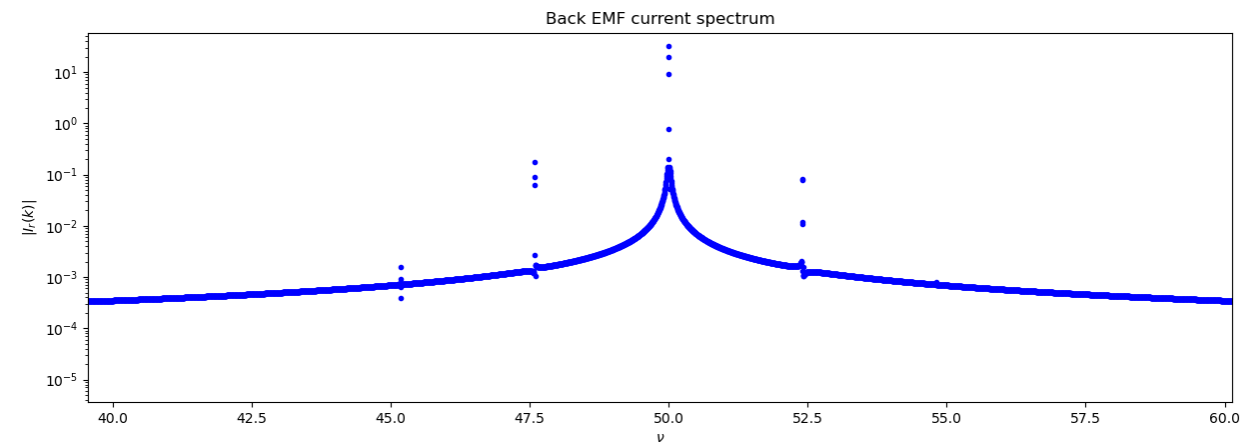

Figure 9. Spectrum of a motor with a defect in one of the rotor loop, $\Phi_{j}=\Phi(1+0.1)$.

tiny spike at $\Omega-4 s$, but this is so small that may be unmeasurable when there is noise in the data. Moreover $\epsilon=0.1$ is quite a significant defect as it means that the maximal flux through that loop has increased by $10 \%$.

\subsubsection{Precession of the rotor}

We can also consider a rotor spinning asymmetrically with a precession frequency $\nu_{\text {pre }}$. This can be modelled using $\Phi_{i}=\Phi\left(1+\epsilon_{\text {pre }} \cos \left(\nu_{\text {pre }} t+\phi_{i}\right)\right)$. When $\nu_{\text {pre }}=0$, the asymmetry rotates at the same speed as the rotor and this is very similar to the asymmetry above, as shown on Figure 10 for $\epsilon_{\text {pre }}=0.03$, which is very similar to the spectrum in Figure 9. Notice that the chosen deformation parameter is quite large as it implies a $3 \%$ difference in the flux due to the wobbling of the rotor. A smaller deformation will results in smaller amplitude in the spectrum.

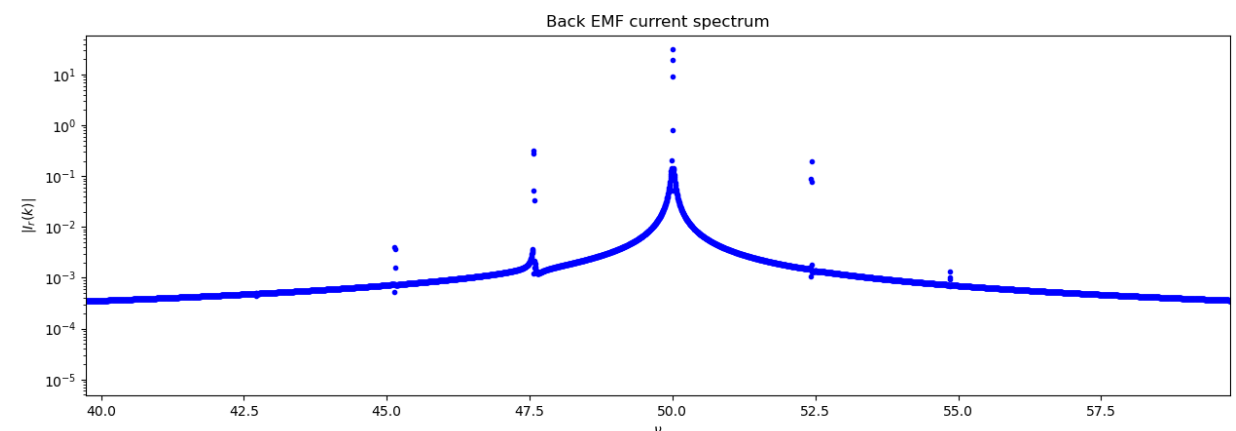

Figure 10. Spectrum of a motor with an asymmetric rotor loop. $\Phi_{i}=\left(1+\epsilon_{\text {pre }} \cos \left(\phi_{i}\right)\right)$, $\epsilon_{\text {pre }}=0.03$, and $\nu_{\text {pre }}=0 \mathrm{~Hz}$.

If the rotor is allowed to precess, i.e. $\nu_{\text {pre }} \neq$, then the spectrum will significantly differ from the previous defects. This can be seen in Figures 11 and 12, both of which show asymmetric spikes about the main frequency $\Omega=50 \mathrm{~Hz}$. Spikes also occur at the 
respective precession frequencies $\nu_{\text {pre }}$ and are surrounded by other spikes separated by the slip frequency.

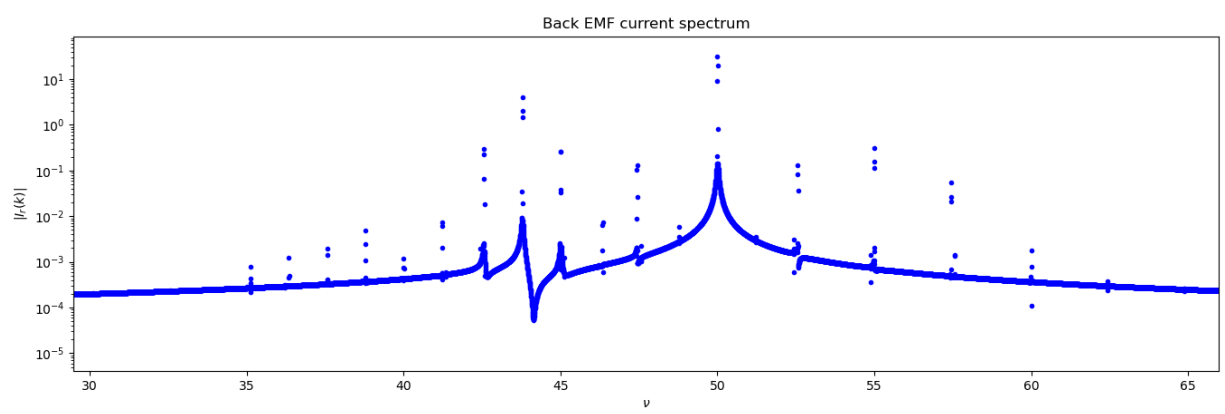

Figure 11. Spectrum of a motor with an asymmetric precessing rotor loop, $\Phi_{i}=(1+$ $\left.\epsilon_{\text {pre }} \cos \left(\nu_{\text {pre }} t+\phi_{i}\right)\right), \epsilon_{\text {pre }}=0.03$, and $\nu_{\text {pre }}=5 \mathrm{~Hz}$.

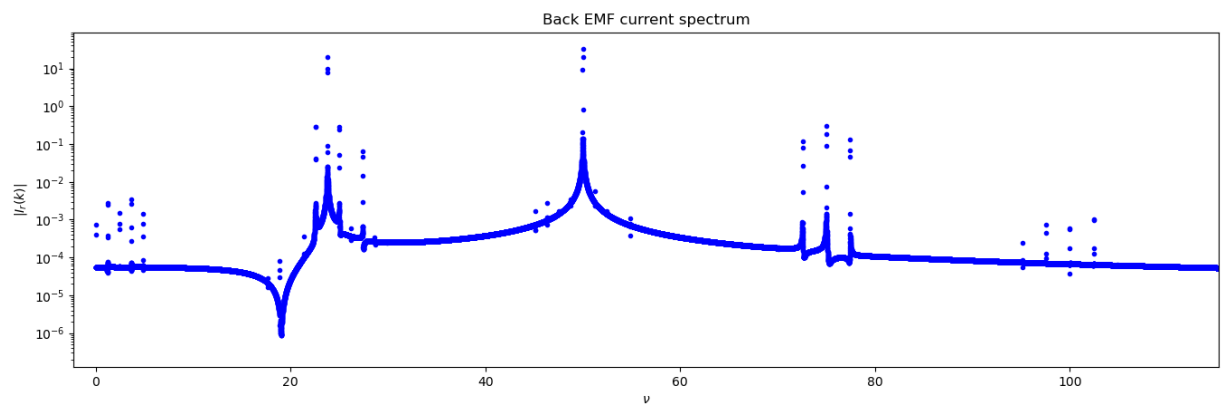

Figure 12. Spectrum of a motor with an asymmetric precessing rotor, $\Phi_{i}=(1+$ $\left.\epsilon_{\text {pre }} \cos \left(\nu_{\text {pre }} t+\phi_{i}\right)\right), \epsilon_{\text {pre }}=0.03$, and $\nu_{\text {pre }}=25 \mathrm{~Hz}$.

\subsubsection{Time variation in the motor load}

The Motor or the load can have a moment of inertia which precesses at frequency $\nu_{I}$. Then we can use $\mathcal{I}=\mathcal{I}_{0}\left(1+\epsilon_{i} \sin \left(\theta-\nu_{i} t\right)\right)$ for some average moment of inertia $\mathcal{I}_{0}$. However numerical results showed that this only modulated the amplitude of the $50 \mathrm{~Hz}$ peak.

The results in this section have all be restricted to a linear model for frictional load. Allowing $\Gamma_{3}$ to be non-zero was seen in numerical experiments to merely change the amplitude of the main peak.

\subsubsection{Time variation in the motor load and load moment of inertia}

The friction parameter $\Gamma$ could also be precessing and be of the form $\Gamma=\Gamma_{0}(1+$ $\left.\epsilon_{\Gamma} \cos \left(\nu_{\Gamma} t\right)\right)$. This would correspond to a load that is precessing. If $\nu=\Omega$, this corresponds to a friction that is $\theta$ dependent inside the load or the bearings. In that case we 
see 2 side peaks around the main's frequency with a frequency different equal to the slip, as shown in Figure 13.

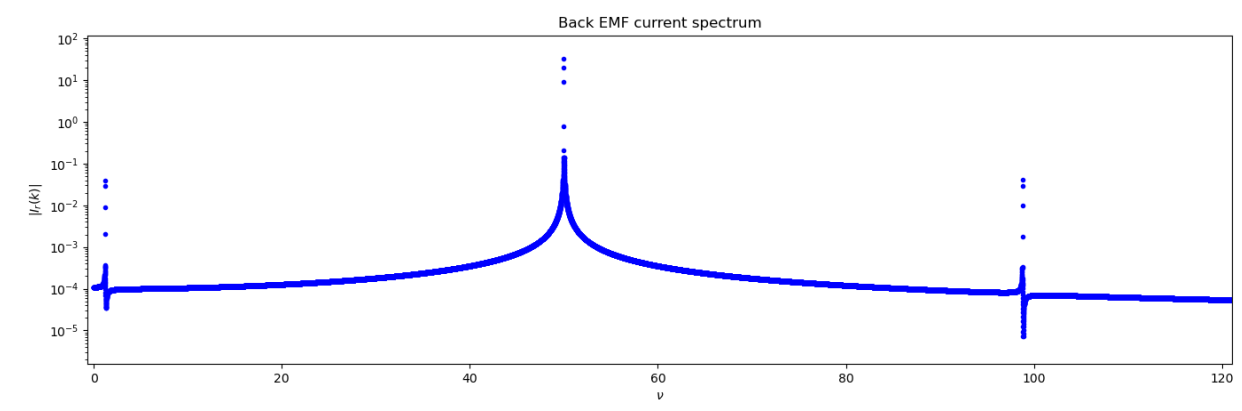

Figure 13. Spectrum of a motor with a time dependant load, $\Gamma=0.2(1+0.1 \cos (\theta(t)))$.

If the load modulation frequency is $5 \mathrm{~Hz}$, then we observe 2 side peaks $5 \mathrm{~Hz}$ away from the main's frequency, as shown on Figure 14.

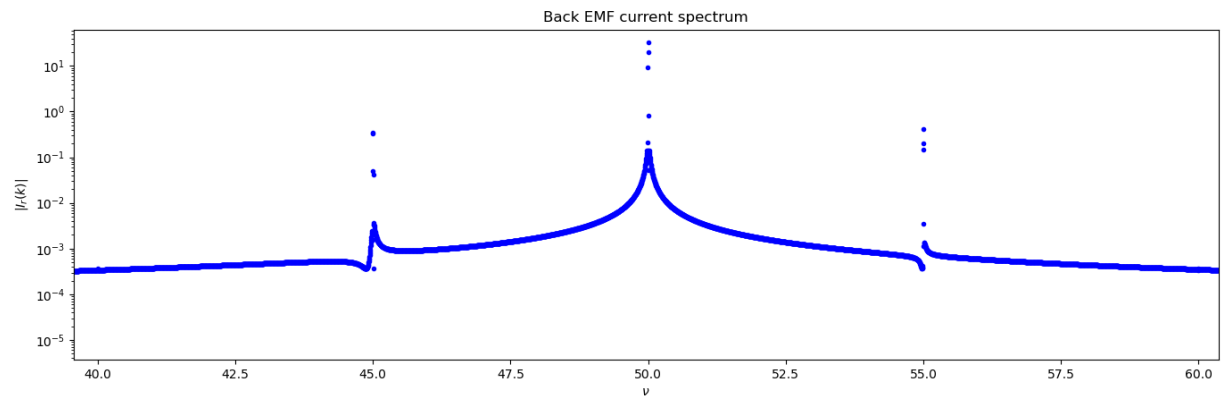

Figure 14. Spectrum of a motor with a time dependant load, $\Gamma=0.2(1+0.1 \sin (2 \pi \nu t))$, $\nu=5 \mathrm{~Hz}$.

Up until now all the periodic perturbations we have considered have been sinusoidal, however we can also consider different periodic functions. If we allow $\Gamma=0.2(1+$ $\left.0.1 \sin ^{3}(2 \pi \nu t)\right)$, we see that further peaks appear in the spectrum shown in Figure 15, all separated by twice the modulation frequency.

We can also consider allowing the periodic perturbation to be dicontinuous, foe example the $S Q R$ function which is one for the first half a period and minus one for the second half period. The resulting spectrum is shown in Figure 16, shows even more peaks than the previous spectrum, all separated by twice the modulation frequency.

\subsubsection{Combined defects}

Finally, it is possible to model the effect of multiple interacting defects. The resulting spectrum is shown in Figure 17, where a single rotor bar is damaged, and both the rotor and frictional load are precessing at different rates. 


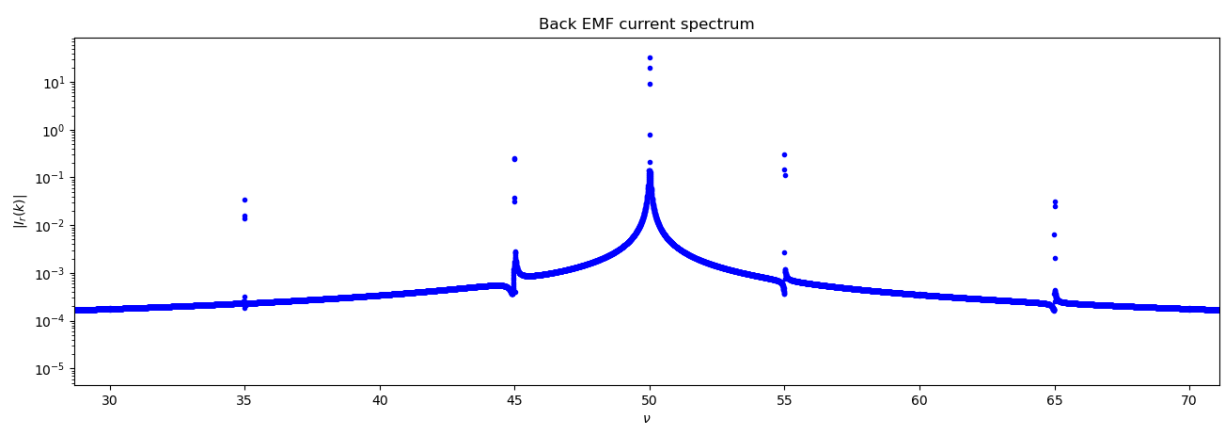

Figure 15. Spectrum of a motor with a time dependant load, $\Gamma=0.2\left(1+0.1 \sin ^{3}(2 \pi \nu t)\right)$, $\nu=5 H z$.

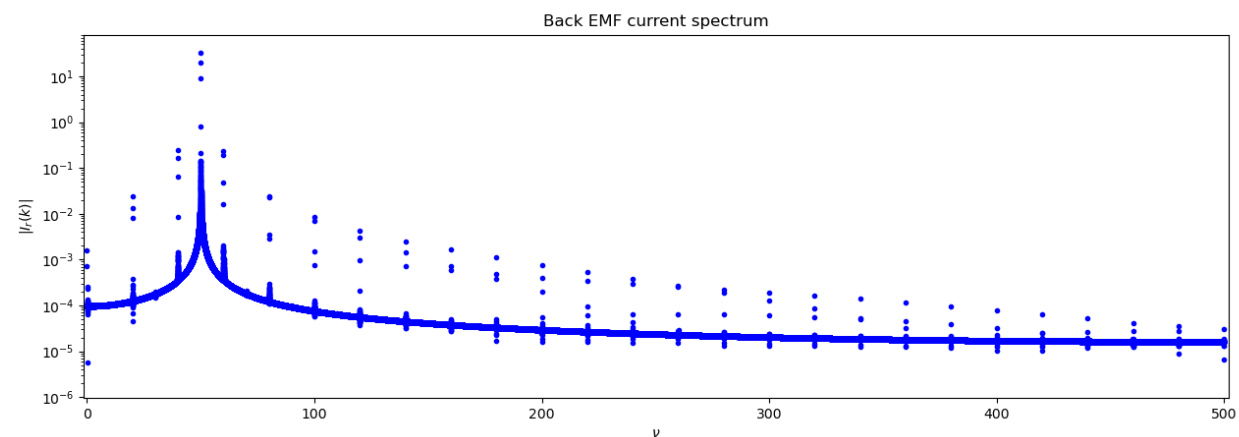

Figure 16. Spectrum of a motor with a time dependant load, $\Gamma=0.2(1+0.1 \mathrm{SQR}(2 \pi \nu t))$, $\nu=10 \mathrm{~Hz}$, where the SQR function is 1 for the first half of the period and -1 for the second.

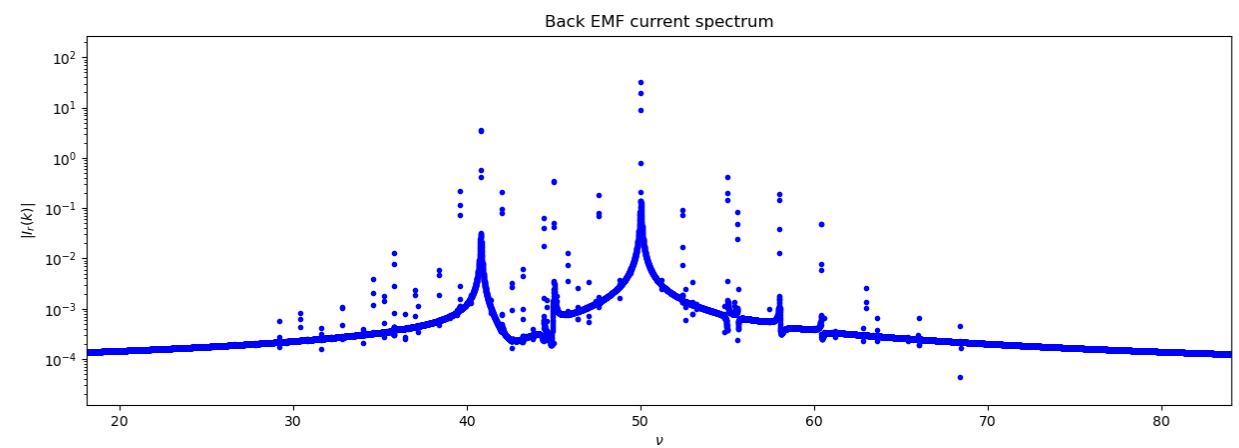

Figure 17. Spectrum of a motor with all modulations combined: $\epsilon=0.1 ; \epsilon_{\text {pre }}=0.02$, $\nu_{\text {pre }}=8 \mathrm{~Hz} ; \epsilon_{\Gamma}=0.1, \nu_{\Gamma}=5 \mathrm{~Hz}$. 


\subsection{Asymptotic results}

In section 5.3 numerical experiments were used to show the effect of various perturbations, and suggested relationships between peak locations and frequencies associated with the problem were given. We will now use asymptotics to rigorously justify the location of peaks associated with particular perturbations.

\subsubsection{Load perturbations}

We consider the simplified case in which the rotor is symmetric and the magnetic field is fixed in time. We shall also assume that the frictional load is linear. Then according to (5.18) and (5.28) the angular position of the rotor and the residual induced current are given by

$$
\begin{aligned}
\mathcal{I} \ddot{\theta} & =-\frac{N \Phi^{2}}{2 R}(\dot{\theta}-\Omega)-\Gamma \dot{\theta}, \\
I_{\text {res }} & =\frac{N}{2} \frac{A_{s} k}{L_{s} R} \Phi(\Omega-\dot{\theta}) \sin (\Omega t),
\end{aligned}
$$

where we have taken $\phi_{s}=0$ for simplicity and the load factor is taken to be

$$
\Gamma=\Gamma_{0}+\epsilon \Gamma_{1} \cos (\psi t),
$$

where $\psi>0$. This is the same form of perturbation as in section 5.3.4.

We expand $\theta, I_{\text {res }}$ in an asymptotic series of the form

$$
\theta=\theta_{0}+\epsilon \theta_{1}+o(\epsilon), \text { and } I_{r e s}=I_{r e s, 0}+\epsilon I_{r e s, 1}+o(\epsilon)
$$

If we assume that the initial conditions for $\theta$ and $\dot{\theta}$ vanish ( the initial data should decay away in long term operation), then the leading order solution is simply

$$
\theta_{0}=\frac{N \Phi^{2} \Omega}{N \Phi^{2}+2 \Gamma_{0} R} t, \text { and } I_{r e s, 0}=N \frac{A_{s} k \Phi}{L_{s} R} \frac{\Gamma_{0} R \Omega}{N \Phi^{2}+2 \Gamma_{0} R} \sin (\Omega t) .
$$

We note that in the limit of no load i.e. $\Gamma_{0} \rightarrow 0$ the residual current goes to zero as expected.

At $\mathcal{O}(\epsilon)$, the angular momentum balance (5.32) gives that

$$
\ddot{\theta}_{1}=-\left(\frac{N \Phi^{2}}{2 R \mathcal{I}}+\frac{\Gamma_{0}}{\mathcal{I}}\right) \dot{\theta}_{1}-\frac{\Gamma_{1}}{\mathcal{I}} \dot{\theta}_{0} \cos (\psi t) .
$$

Again, assuming the complementary function is negligible (on long timescale), we seek the particular solution, and find that

$$
\theta_{1}=\frac{\Gamma_{1} \dot{\theta}_{0}}{\mathcal{I}\left(\psi^{2}+\xi^{2}\right)} \cos (\psi t)-\frac{\xi \Gamma_{1} \dot{\theta}_{0}}{\psi \mathcal{I}\left(\psi^{2}+\xi^{2}\right)} \sin (\psi t),
$$

where $\xi=N \Phi^{2} /(2 R \mathcal{I})+\Gamma_{0} / \mathcal{I}$. Therefore, by considering $(5.33)$ at $\mathcal{O}(\epsilon)$, we find that 
the residual current associated with this perturbation is

$$
\begin{aligned}
I_{r e s, 1}=-\frac{N}{4} \frac{A_{s} K \Phi}{L_{s} R} \frac{\Gamma_{1} \dot{\theta}_{0}}{\mathcal{I}\left(\psi^{2}+\xi^{2}\right)}(\sin ((\Omega+\psi) t)+\sin ((\Omega-\psi) t) \\
\\
\quad-\frac{\xi}{\psi} \cos ((\Omega-\psi) t)+\frac{\xi}{\psi} \cos ((\Omega+\psi) t)
\end{aligned}
$$

Thus we have found the sideband frequencies to be at $\Omega \pm \psi$, as well as an estimate for the size of the residual current. This agree's with the numerical findings in Figure 14, which found sidebands $5 \mathrm{~Hz}$ away from the main band when a load perturbation of $5 \mathrm{~Hz}$ was applied.

\subsubsection{Load and magnetic flux perturbations}

An interesting future problem would be to consider both load and magnetic flux perturbations. For simplicity, we shall assume that all of the $\Phi_{i}=\Phi$, a constant except for $\Phi_{1}=\Phi+\epsilon \tilde{\Phi}$, where $\tilde{\Phi}=\tilde{\Phi}_{0} \cos \left(\psi_{1}\right)$. As in the previous section we assume that the frictional load is linear, with $\Gamma$ given by $\Gamma=\Gamma_{0}+\epsilon \tilde{\Gamma}$, where $\tilde{\Gamma}=\tilde{\Gamma}_{0} \cos \left(\psi_{2} t\right)$. We also assume that $k_{i}=k$ for all $i$ and that $\phi_{s}=0$ for simplicity.Substituting these expressions into (5.15) and (5.27) we obtain the following governing equations for $\theta$ and $I_{\text {res }}$

$$
\begin{aligned}
\mathcal{I} \ddot{\theta} & =\epsilon \frac{\Phi \dot{\tilde{\Phi}}}{2 R} \sin (2(\theta-\Omega t))-(\dot{\theta}-\Omega) \frac{\Phi}{R}\left(\frac{N \Phi}{2}+2 \epsilon \tilde{\Phi} \sin ^{2}(\theta-\Omega t)\right)-\Gamma_{0} \dot{\theta}-\epsilon \tilde{\Gamma}+\mathcal{O}\left(\epsilon^{2}\right), \\
I_{\text {res }} & =\frac{N}{2} \frac{A_{s} k}{L_{s} R} \Phi(\Omega-\dot{\theta}) \sin (\Omega t)-\frac{\epsilon}{2} \frac{A_{s} k}{L_{s} R} \tilde{\Phi}(\Omega-\dot{\theta})(\sin (\Omega t)+\sin (\Omega t-2 \theta))+\mathcal{O}\left(\epsilon^{2}\right) .
\end{aligned}
$$

We expand $\theta$ and $I_{\text {res }}$ as asymptotic series of the form $\theta=\theta_{0}+\epsilon \theta_{1}+o(\epsilon)$, and $I_{\text {res }}=$ $I_{\text {res }, 0}+\epsilon I_{\text {res }, 1}+o(\epsilon)$. We find that the leading order problem is identical to that from section 5.4.1, and hence has the same particular solution given by (5.36).

At $\mathcal{O}(\epsilon)$ we find that

$$
\mathcal{I} \ddot{\theta}_{1}=\frac{\Phi \dot{\tilde{\Phi}}}{2 R} \sin \left(2\left(\theta_{0}-\Omega t\right)\right)-2\left(\dot{\theta}_{0}-\Omega\right) \frac{\Phi}{R} \tilde{\Phi} \sin ^{2}\left(\theta_{0}-\Omega t\right)-\dot{\theta}_{1} \frac{\Phi}{R} \frac{N \Phi}{2}-\Gamma_{0} \dot{\theta}_{1}-\tilde{\Gamma} \dot{\theta}_{0}
$$

After substituting in $\tilde{\Phi}$ and $\tilde{\Gamma}$, and defining $\xi=\Phi^{2} N /(2 R \mathcal{I})$ this becomes

$$
\begin{aligned}
\ddot{\theta}_{1}+\xi \dot{\theta}_{1}=-\frac{\Phi \tilde{\Phi}_{0} \psi_{1}}{2 R} & \sin \left(2\left(\theta_{0}-\Omega t\right)\right) \sin \left(\psi_{1} t\right) \\
& -2 \frac{\Phi \tilde{\Phi}_{0}}{R}\left(\dot{\theta}_{0}-\Omega\right) \sin ^{2}\left(\theta_{0}-\Omega t\right) \cos \left(\psi_{1} t\right)-\frac{\tilde{\Gamma}_{0}}{\mathcal{I}} \dot{\theta}_{0} \cos \left(\psi_{2} t\right) .
\end{aligned}
$$


We apply trigonometric identities to rewrite this as

$$
\begin{array}{r}
\ddot{\theta}_{1}+\xi \dot{\theta}_{1}=-\frac{\Phi \tilde{\Phi}_{0} \psi_{1}}{4 R}\left(\cos \left(\left(2 \dot{\theta}_{0}-2 \Omega-\psi_{1}\right) t\right)-\cos \left(\left(2 \dot{\theta}_{0}-2 \Omega+\psi_{1}\right) t\right)\right) \\
-\frac{\Phi \tilde{\Phi}_{0}}{2 R}\left(\dot{\theta}_{0}-\Omega\right)\left(-\cos \left(\left(2 \dot{\theta}_{0}-2 \Omega-\psi_{1}\right) t\right)-\cos \left(\left(2 \dot{\theta}_{0}-2 \Omega+\psi_{1}\right) t\right)+2 \cos \left(\psi_{1} t\right)\right) \\
-\frac{\tilde{\Gamma}_{0}}{\mathcal{I}} \dot{\theta}_{0} \cos \left(\psi_{2} t\right) .
\end{array}
$$

Hence the particular solution we seek should by of the form

$$
\begin{aligned}
\theta_{1}=C_{1} \cos \left(\psi_{1} t\right) & +C_{2} \sin \left(\psi_{1} t\right)+C_{3} \cos \left(\psi_{2} t\right)+C_{4} \sin \left(\psi_{2} t\right) \\
+C_{5} & \cos \left(\left(2 \dot{\theta}_{0}-2 \Omega-\psi_{1}\right) t\right)+C_{6} \sin \left(\left(2 \dot{\theta}_{0}-2 \Omega-\psi_{1}\right) t\right) \\
& +C_{7} \cos \left(\left(2 \dot{\theta}_{0}-2 \Omega+\psi_{1}\right) t\right)+C_{8} \sin \left(\left(2 \dot{\theta}_{0}-2 \Omega+\psi_{1}\right) t\right),
\end{aligned}
$$

where $C_{1}, C_{2} \ldots, C_{8}$ are real constants. While finding the coefficients is a nontrivial algebraic exercise, we can use the functional form of (5.45) to find the excited frequencies of $I_{r e s, 1}$. At $\mathcal{O}(\epsilon)$ we find that

$$
\begin{aligned}
I_{r e s, 1}=-\frac{N}{2} & \frac{A_{s} k}{L_{s} R} \Phi \sin (\Omega t) \dot{\theta}_{1} \\
& \quad-\frac{1}{2} \frac{A_{s} k}{L_{s} R} \tilde{\Phi}_{0}\left(\Omega-\dot{\theta}_{0}\right)\left(\cos \left(\psi_{1} t\right) \sin (\Omega t)+\cos \left(\psi_{1} t\right) \sin \left(\Omega t-2 \theta_{0}\right)\right) .
\end{aligned}
$$

If we apply trigonometric identities then the final form of $I_{\text {res }, 1}$ will be

$$
\begin{gathered}
I_{r e s, 1}=\hat{C}_{1} \cos \left(\left(\Omega+\psi_{1}\right) t\right)+\hat{C}_{2} \cos \left(\left(\Omega-\psi_{1}\right) t\right)+\hat{C}_{3} \sin \left(\left(\Omega+\psi_{1}\right) t\right)+\hat{C}_{4} \sin \left(\left(\Omega-\psi_{1}\right) t\right) \\
\hat{C}_{5} \cos \left(\left(\Omega+\psi_{2}\right) t\right)+\hat{C}_{6} \cos \left(\left(\Omega-\psi_{2}\right) t\right)+\hat{C}_{7} \sin \left(\left(\Omega+\psi_{2}\right) t\right)+\hat{C}_{8} \sin \left(\left(\Omega-\psi_{2}\right) t\right) \\
+\hat{C}_{9} \cos \left(\left(\Omega-2 \dot{\theta}_{0}+\psi_{1}\right) t\right)+\hat{C}_{10} \cos \left(\left(\Omega-2 \dot{\theta}_{0}-\psi_{1}\right) t\right) \\
\quad+\hat{C}_{11} \cos \left(\left(3 \Omega-2 \dot{\theta}_{0}+\psi_{1}\right) t\right)+\hat{C}_{12} \cos \left(\left(3 \Omega-2 \dot{\theta}_{0}-\psi_{1}\right) t\right), \quad
\end{gathered}
$$

where $\hat{C}_{1}, \ldots, \hat{C}_{12}$ are constants that could be determined. This tells us that the excited frequencies at $\mathcal{O}(\epsilon)$ are

$$
\left|\Omega \pm \psi_{1}\right|,\left|\Omega \pm \psi_{2}\right|,\left|\Omega-2 \dot{\theta}_{0} \pm \psi_{1}\right|, \text { and }\left|3 \Omega-2 \dot{\theta}_{0} \pm \psi_{1}\right| \text {. }
$$

We take the absolute value as some of the expressions may be negative. We note that the final four frequencies are asymmetric about $\Omega$.

\section{Effects from the driven rotating machinery}

In the previous section we have developed a physical for an induction motor and studied its behaviour under various perturbations. However, these perturbations have been restricted to effects taking place within the motor; in reality the behaviour of the mechanical machine driven by the motor will also perturb the motor. In this section we discuss how these effects could be incorporated into a model for residual current. 


\subsection{Disc driven directly by the motor}

Consider the situation shown in Figure 18 (a), where a disc is directly connected to the motor which applies torque at its center CD, and is supported by vertical and lateral springs with stiffnesses $k_{v}$ and $k_{u}$ respectively. The rotor has diametral moment of inertia $I_{d}$ and mass $M$. Due to slight imperfections in its geometry, the centre of mass is located at point CG as shown in Figure 18 (b), at a distance $\epsilon$ from CD, and this leads to out of balance forces as it spins. The out of balance is assumed to be along the horizontal axis for $\theta=0$ but this causes no loss in generality.

The kinetic energy is given by

$$
T=\frac{I_{d}}{2} \dot{\theta}^{2}+\frac{M}{2}\left((\dot{u}-\dot{\theta} \sin \theta)^{2}+(\dot{v}+\dot{\theta} \cos \theta)^{2}\right),
$$

and the potential energy by

$$
V=\frac{k_{u}}{2} u^{2}+\frac{k_{v}}{2} v^{2}
$$

This leads to the following equations of motion through the usual Lagrangian process:

$$
\begin{aligned}
M \ddot{u}+c_{x} \dot{u}+k_{x} u & =\epsilon M\left(\dot{\theta}^{2} \cos \theta+\ddot{\theta} \sin \theta\right)+\text { contact terms }, \\
M \ddot{v}+c_{y} \dot{v}+k_{y} v & =\epsilon M\left(\dot{\theta}^{2} \sin \theta-\ddot{\theta} \cos \theta\right)+\text { contact terms, } \\
{\left[I_{d}+\epsilon^{2} M\right] \ddot{\theta}+c_{\theta} \dot{\theta} } & =\epsilon M(\ddot{u} \sin \theta-\ddot{v} \cos \theta)+\text { motor torque }+ \text { contact moments. }
\end{aligned}
$$

where linear damping terms have also been included, in addition to place holders for the dynamics arising from the motor itself, and disturbances from nonlinearities, faults, rubs etc.

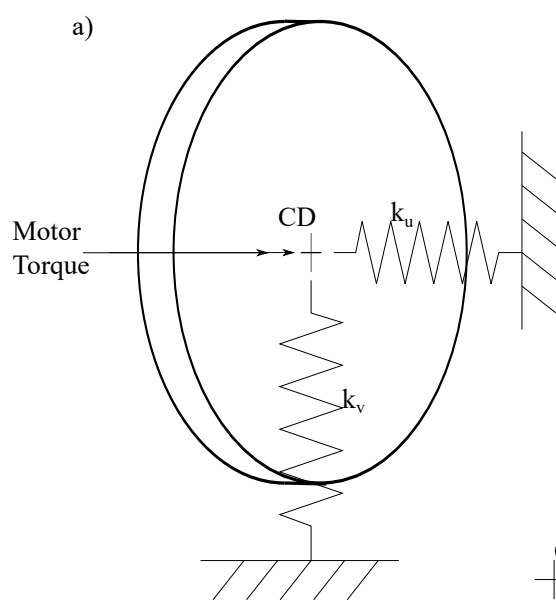

b)

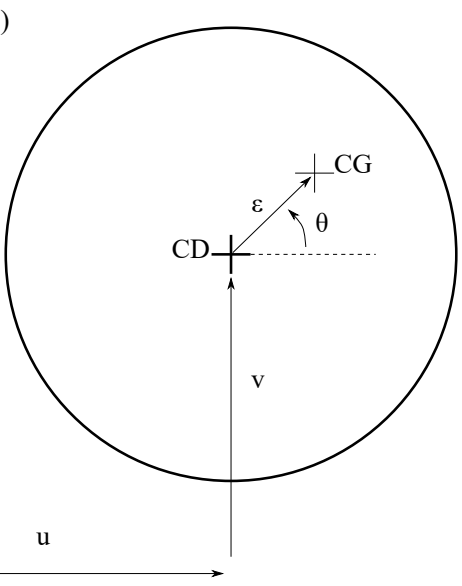

Figure 18. a) A disc with torque applied by the motor at its centre supported by lateral spring supports. b) Degrees of freedom used in analysis.

If we assume the motor to have a symmetric rotor with no defects then by (5.18) the 
equations of motion become

$$
\begin{aligned}
M \ddot{u}+c_{x} \dot{u}+k_{x} u & =\epsilon M\left(\dot{\theta}^{2} \cos \theta+\ddot{\theta} \sin \theta\right)+\mathcal{C}_{x}, \\
M \ddot{v}+c_{y} \dot{v}+k_{y} v & =\epsilon M\left(\dot{\theta}^{2} \sin \theta-\ddot{\theta} \cos \theta\right)+\mathcal{C}_{y}, \\
{\left[I_{d}+\epsilon^{2} M\right] \ddot{\theta}+c_{\theta} \dot{\theta} } & =\epsilon M(\ddot{u} \sin \theta-\ddot{v} \cos \theta)+\frac{N \Phi^{2}}{2 R}(\Omega-\dot{\theta})+\mathcal{C}_{\theta},
\end{aligned}
$$

where $\mathcal{C}_{x}, \mathcal{C}_{y}$, and $\mathcal{C}_{\theta}$ are the contact terms in the respective equations.

A method to numerically solve (6.4) is given in section B. Some preliminary results of calculating the resulting spectrum fom solving this using (5.28) are given in Figure 19.

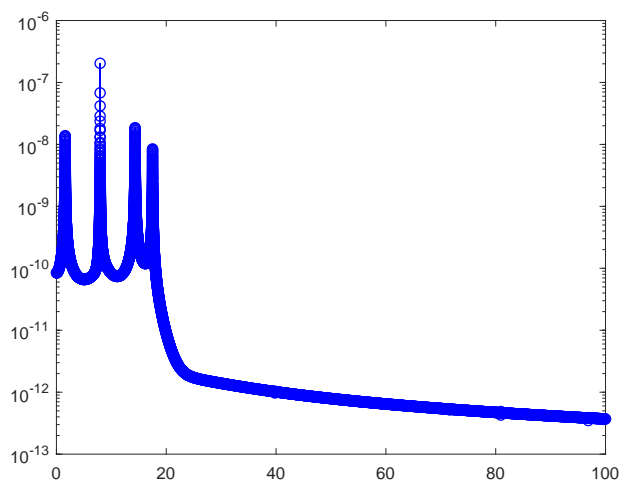

Figure 19. Preliminary result of finding spectrum of a rotor coupled to spinning disc.

\subsection{A flexible rotor shaft on rigid supports}

An alternative system that could be driven is a more flexible machine, where the rotor is supported on a long shaft $L$. This can be modelled with the same degrees of freedom as the previous example if a single spring approximation is made for the shaft, giving $k_{u}=k_{v}=\frac{48 E I}{L^{3}}$, and rotational degrees of freedom are still neglected. In a purely lateral model neglecting torsion and assuming a constant drive speed, this model would be very similar in behaviour to the directly driven motor, however when considering lateral-torsion couplings additional terms appear in the flexible system. The key difference between the systems, is that with reference to Figure 18 (b), in the directly driven system the torque is applied at CD, whereas in the flexible rotor, the motor torque is applied at $\mathrm{O}$, and therefore further moments arise due to displacements $u$ and $v$.

We can consider the effect of these by evaluating the angular inertia about $\mathrm{O}$, given by $\mathbf{x} \times \dot{\mathbf{x}}$, where $\mathbf{x}=[u+\epsilon \cos \theta, v+\epsilon \sin \theta]^{T}$, and differentiating the result with regard to time. The torque provided by the motor must be the rate of change of angular inertia given by:

$$
M\left(u \ddot{v}-\ddot{u} v+\epsilon^{2} \ddot{\theta}+\epsilon\left(u \ddot{\theta}+\ddot{v}+v \dot{\theta}^{2}\right) \cos \theta+\epsilon\left(v \ddot{\theta}-\ddot{u}-u \dot{\theta}^{2}\right) \sin \theta\right)
$$




\section{Motor Torque}

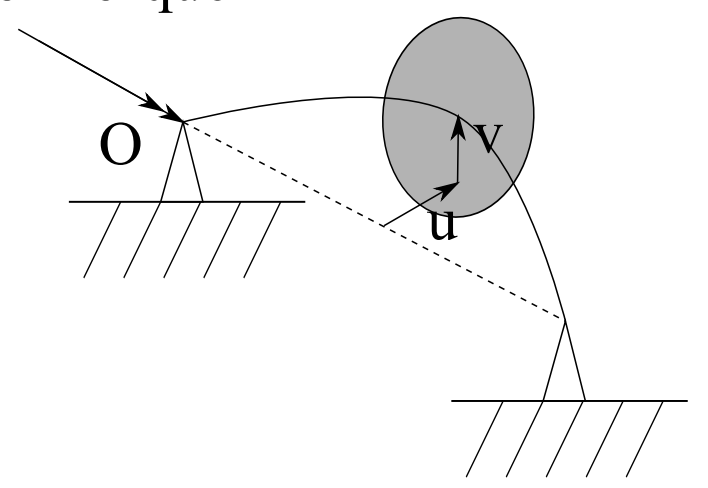

Figure 20. a) A disc with torque applied by the motor at its centre supported by lateral spring supports. b) Degrees of freedom used in analysis.

Hence the equations in this case become:

$$
\begin{aligned}
M \ddot{u}+c_{x} \dot{u}+k_{x} u & =\epsilon M\left(\dot{\theta}^{2} \cos \theta+\ddot{\theta} \sin \theta\right)+\mathcal{C}_{x} \\
M \ddot{v}+c_{y} \dot{v}+k_{y} v & =\epsilon M\left(\dot{\theta}^{2} \sin \theta-\ddot{\theta} \cos \theta\right)+\mathcal{C}_{y} \\
{\left[I_{d}+\epsilon^{2} M\right] \ddot{\theta}+c_{\theta} \dot{\theta} } & =\epsilon M(\ddot{u} \sin \theta-\ddot{v} \cos \theta)+\frac{N \Phi^{2}}{2 R}(\Omega-\dot{\theta}) \\
& -\epsilon M\left[u\left(\dot{\theta}^{2} \sin \theta-\ddot{\theta} \cos \theta\right)-v\left(\dot{\theta}^{2} \cos (\theta)+\ddot{\theta} \sin \theta\right)\right] \\
& -M(v \ddot{u}-u \ddot{v})+\mathcal{C}_{\theta} .
\end{aligned}
$$

Note that the extra coupling terms seem to give a greater sensitivity to the lateral/torsional coupling, because they are on the order of $u$ and $v$, which depending on the proximity to a resonant drive speed and the flexibility of the shaft, could be significantly greater than $\epsilon$. In principle a Lagrangian approach where the moments of inertia vary due to a parallel axis theorem effect due to $u$ and $v$ should give a similar result. For more details on how to derive appropriate equations of motion, see e.g. [12]. 


\section{Conclusions and further directions}

In this report we have discussed two models for an electric motor, the Steinmetz equivalent circuit in section 4 and a physics based model based on Faraday's law of induction, Ohm's law, and balance of angular momentum derived in section 5. We have shown that both models are capable of predicting sidebands in the residual current spectrum, based on prescribed perturbations to various parameters. The Steinmetz model has the advantaged of being established in electrical engineering and hence is easy to parameterise. However the rotor dynamics $\theta(t)$ only enter the model indirectly through the slip parameter, and we were only able to produce symmetric sidebands using sinusoidal perturbations. In contrast, the physical laws based model predicts both rotor dynamics $\theta(t)$ and the residual current $I_{\text {res }}$. We have shown how this model can be used to predict the spectrum of a motor with either a damaged bar, a precessing rotor, or a time varying load. In section 6 we have shown, through simple examples, how the physical laws model can be coupled into a larger model for the machinery being driven by the motor, allowing us to quantify the effect of perturbations on the load driven by lateral vibrations. In particular, we have shown numerically that asymmetric sidebands can be produced by a precessing rotor, and confirmed this prediction asymptotically. The disadvantage of the physical model is that it has more parameters that must be found estimated, or measured.

Although the work here is a good basis for a model of an induction motor, more work is required to fully explain the behaviour of the residual current to specific perturbations in particular:

- A literature review for torque models in other parts of the engineering literature that have not been applied to current signature analysis.

- Numerical results with realistic parameters allowing a more insightful comparison of the asymptotic and numerical approaches, and if possible experimental data.

- Further development om coupling the rotor model to a model for external machinery.

- In particular can we distinguish between harmonics due to motor faults and due to contact or rub? What is the signature of a rub or contact nonlinearity in the motor current signal that is being monitored?

- Examining the inverse problem. How practical is it to use these physical models in the data processing of a given signal.

There is also an interesting mathematical problem of the correct continuum model as the number of wire loops in the rotor becomes very large. Recent studies on Faraday cages have shown the coupling between adjacent wires can have important effects on the marcroscale behaviour of electrical devices.

\section{References}

[1] Faraday's law of induction. https://en.wikipedia.org/wiki/Faraday's_law_ of_induction, January 2021.

[2] The Feymann lectures on physics vol. ii ch. 22: AC circuits. https://www. feynmanlectures.caltech.edu/II_22.html, January 2021. 
[3] Inductance. https://en.wikipedia.org/wiki/Inductance, January 2021.

[4] Induction motor. https://en.wikipedia.org/wiki/Induction_motor, January 2021. date accessed $21 / 04 / 2001$.

[5] Lorentz force. https://en.wikipedia.org/wiki/Lorentz_force, January 2021.

[6] A. Aggarwal, E.G. Strangas, and J. Agapiou. Analysis of unbalanced magnetic pull in pmsm due to static eccentricity. In 2019 IEEE Energy Conversion Congress and Exposition (ECCE), pages 4507-4514. IEEE, 2019.

[7] A. Bouras, S. Bennedjai, and S. Bouras. Experimental detection of defects in variable speed fan bearing using stator current monitoring. SN Applied Sciences, 2(5):1-8, 2020.

[8] J.R. Cameron, W.T. Thomson, and A. B. Dow. Vibration and current monitoring for detecting airgap eccentricity in large induction motors. In IEE Proceedings $B$ (Electric Power Applications), volume 133, pages 155-163. IET, 1986.

[9] S. J. Chapman, D.P. Hewett, and L. N. Trefethen. Mathematics of the faraday cage. Siam Review, 57(3):398-417, 2015.

[10] J. Cusido, J. Rosero, E. Aldabas, J. A. Ortega, and L. Romeral. New fault detection techniques for induction motors. Electrical Power quality and utilisation, Magazine, 2(1), 2006.

[11] J. Faiz, B. M. Ebrahimi, B. Akin, and H. A. Toliyat. Dynamic analysis of mixed eccentricity signatures at various operating points and scrutiny of related indices for induction motors. IET Electric Power Applications, 4(1):1-16, 2010.

[12] M. Friswell, K. Penny, S. Garvey, and A. Lees. Fundamentals of Rotor Dynamics. Cambridge University Press, 2010.

[13] A. Ghoggalm, S.E. Zouzou, H. Razik, M. Sahraoui, and A. Khezzar. An improved model of induction motors for diagnosis purposes-slot skewing effect and air-gap eccentricity faults. Energy conversion and Management, 50(5):1336-1347, 2009.

[14] M. E. Halpin. Listening to the murmur of electric motors. Final year dissertation, University of Durham, 2020.

[15] D. P. Hewett and I. J. Hewitt. Homogenized boundary conditions and resonance effects in faraday cages. Proceedings of the Royal Society A: Mathematical, Physical and Engineering Sciences, 472(2189):20160062, 2016.

[16] S. Jurkovic. Induction motor parameters extraction. Educypedia-Electronics, 2014.

[17] V.C.M.N. Leite, J. G.B. da Silva, G.F.C. Veloso, L.E.B. da Silva, G. LambertTorres, E.L. Bonaldi, and L. E. E. de Oliveira. Detection of localized bearing faults in induction machines by spectral kurtosis and envelope analysis of stator current. IEEE Transactions on Industrial Electronics, 62(3):1855-1865, 2014.

[18] H. D. Lidford. Signal analysis in mechanical engines. Final year dissertation, University of Durham, 2020.

[19] N. Marrin. Investigating nonlinear phenomena in a rotor model with hydrodynamic journal bearings. Msc thesis, University of Bristol, 2020.

[20] M. R. Mehrjou, N. Mariun, M. H. Marhaban, and N. Misron. Rotor fault condition monitoring techniques for squirrel-cage induction machine - a review. Mechanical Systems and Signal Processing, 25(8):2827-2848, 2011.

[21] S. Nandi. Modeling of induction machines including stator and rotor slot effects. In 38th IAS Annual Meeting on Conference Record of the Industry Applications 
Conference, 2003., volume 2, pages 1082-1089. IEEE, 2003.

[22] S. Nandi, S. Ahmed, and H. A. Toliyat. Detection of rotor slot and other eccentricity related harmonics in a three phase induction motor with different rotor cages. IEEE Transactions on Energy Conversion, 16(3):253-260, 2001.

[23] S. Nandi, R. M. Bharadwaj, and H. A. Toliyat. Performance analysis of a threephase induction motor under mixed eccentricity condition. IEEE Transactions on Energy Conversion, 17(3):392-399, 2002.

[24] P. Pennacchi. Nonlinear effects due to electromechanical interaction in generators with smooth poles. Nonlinear Dynamics, 57(4):607-622, 2009.

[25] A.A. Salah, D.G. Dorrell, and Y. Guo. A review of the monitoring and damping unbalanced magnetic pull in induction machines due to rotor eccentricity. IEEE Transactions on Industry Applications, 55(3):2569-2580, 2019.

[26] L. F. Shampine and M. W. Reichelt. The Matlab ODE suite. SIAM journal on scientific computing, 18(1):1-22, 1997.

[27] W. T. Thomson and M. Fenger. Current signature analysis to detect induction motor faults. IEEE Industry Applications Magazine, 7(4):26-34, 2001.

[28] P. Zhang, Y. Du, T.G. Habetler, and B. Lu. A survey of condition monitoring and protection methods for medium-voltage induction motors. IEEE Transactions on Industry Applications, 47(1):34-46, 2010.

\section{Appendix A Deriving and solving the Steinmetz ODE system}

\section{A.1 Derivation}

By substituting (4.8) into (4.10) we eliminate $I_{r}^{\prime}$, leaving

$$
\begin{array}{r}
R_{\mathrm{rat}} I_{s}+(X / R)_{s, r} \dot{I}_{s}-(X / R)_{m, r} \dot{I}_{m}=V, \\
\left((X / R)_{r, r}+(X / R)_{m, r}\right) s \dot{I}_{m}+(X / R)_{r, r} s \dot{I}_{s}+I_{s}+I_{m}=0 .
\end{array}
$$

Next we rearrange (A 2) for $\dot{I}_{m}$ and substitute it into (A 1)

$$
\begin{gathered}
\left((X / R)_{m, r}(X / R)_{r, r}+(X / R)_{m, r}(X / R)_{s, r}+(X / R)_{r, r}(X / R)_{s, r}\right) s \dot{I}_{s} \\
+\left(\left((X / R)_{m, r}+(X / R)_{r, r}\right) R_{\mathrm{rat}} s+(X / R)_{m, r}\right) I_{s}+(X / R)_{m, r} I_{m} \\
=\left((X / R)_{m, r}+(X / R)_{r, r}\right) s V .
\end{gathered}
$$

In the general case where $s$ is a function of time, differentiating gives

$$
\begin{aligned}
& \quad\left((X / R)_{m, r}(X / R)_{r, r}+(X / R)_{m, r}(X / R)_{s, r}+(X / R)_{r, r}(X / R)_{s, r}\right) s \ddot{I}_{s} \\
& \quad+\left((X / R)_{m, r}(X / R)_{r, r}+(X / R)_{m, r}(X / R)_{s, r}+(X / R)_{r, r}(X / R)_{s, r}\right) \dot{s} \dot{I}_{s} \\
& \left.\quad+\left((X / R)_{m, r}+(X / R)_{r, r}\right) R_{\mathrm{rat}} s+(X / R)_{m, r}\right) \dot{I}_{s}+\left((X / R)_{m, r}+(X / R)_{r, r}\right) R_{\mathrm{rat}} \dot{s} I_{s} \\
& \quad+(X / R)_{m, r} \dot{I}_{m}=\left((X / R)_{m, r}+(X / R)_{r, r}\right) s \dot{V}+\left((X / R)_{m, r}+(X / R)_{r, r}\right) \dot{s} V .
\end{aligned}
$$


We can then substitute in (A 2) again to eliminate $\dot{I}_{m}$,

$$
\begin{gathered}
\left((X / R)_{m, r}(X / R)_{r, r}+(X / R)_{m, r}(X / R)_{s, r}+(X / R)_{r, r}(X / R)_{s, r}\right) s \ddot{I}_{s} \\
+\left((X / R)_{m, r}(X / R)_{r, r}+(X / R)_{m, r}(X / R)_{s, r}+(X / R)_{r, r}(X / R)_{s, r}\right) \dot{s} \dot{I}_{s} \\
+\left(\left((X / R)_{m, r}+(X / R)_{r, r}\right) R_{\mathrm{rat}} s+(X / R)_{m, r}\right) \dot{I}_{s}+\left((X / R)_{m, r}+(X / R)_{r, r}\right) R_{\mathrm{rat}} \dot{s} I_{s} \\
+R_{\mathrm{rat}} I_{s}+(X / R)_{s, r} \dot{I}_{s} \\
\left.=\left((X / R)_{m, r}+(X / R)_{r, r}\right) s \dot{V}+\left((X / R)_{m, r}+(X / R)_{r, r}\right) \dot{s} V+V . \quad \mathrm{A} 5\right) \\
{\left[\left((X / R)_{m, r}(X / R)_{r, r}+(X / R)_{m, r}(X / R)_{s, r}+(X / R)_{r, r}(X / R)_{s, r}\right) s\right] \ddot{I}} \\
+\left[(X / R)_{m, r}(X / R)_{r, r}+(X / R)_{m, r}(X / R)_{s, r}+(X / R)_{r, r}(X / R)_{s, r}\right) \dot{s} \\
\left.+\left((X / R)_{m, r}+(X / R)_{r, r}\right) R_{\mathrm{rat}} s+(X / R)_{m, r}+(X / R)_{s, r}\right] \dot{I}_{s} \\
+\left[\left((X / R)_{m, r}+(X / R)_{r, r}\right) R_{\mathrm{rat}} \dot{s}+R R_{\mathrm{rat}}\right] I_{s} \\
=\left((X / R)_{m, r}+(X / R)_{r, r}\right) s \dot{V}+\left((X / R)_{m, r}+(X / R)_{r, r}\right) \dot{s} V+V . \quad(\mathrm{A} 6)
\end{gathered}
$$

To recover the ODE for the case of constant slip we set $\dot{s}=0$ in (A 6 )

\section{A.2 Numerical solution}

To solve (4.15) we rewrite it as a first order system, with variable $\mathbf{u}=\left(u_{1}, u_{2}\right)$ where $u_{1}=I_{s}$ and $u_{2}=\dot{I}_{s}$. Then the system is

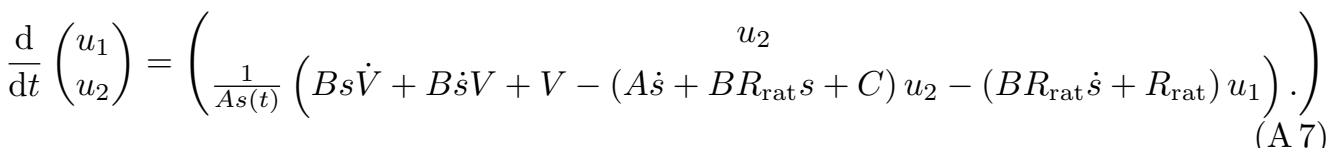

This is then solved using Matlab's built in ODE solver ode45 [26]. To solve the constant slip case, (A 7 ) is used with $\dot{s}=0$.

\section{Appendix B Numerical solution of driven machinery problem}

We will solve for $u, v, \theta$ by rewriting the problems as first order systems and use MATLAB's built-in ODE solvers. To do this we introduce the variable

$$
\overrightarrow{\mathcal{W}}=\left(\mathcal{W}_{1}, \mathcal{W}_{2}, \mathcal{W}_{3}, \mathcal{W}_{4}, \mathcal{W}_{5}, \mathcal{W}_{6}\right)=(u, v, \theta, \dot{u}, \dot{v}, \dot{\theta})
$$




\section{B.1 Numerical solution of disc driven by motor}

The equation of motion for a driven disc (6.4) becomes

$$
\begin{aligned}
\dot{\mathcal{W}}_{1} & =\mathcal{W}_{4}, \\
\dot{\mathcal{W}}_{2} & =\mathcal{W}_{5}, \\
\dot{\mathcal{W}}_{3} & =\mathcal{W}_{6}, \\
M \dot{\mathcal{W}}_{4}-\epsilon M \sin \left(\mathcal{W}_{3}\right) \dot{\mathcal{W}}_{6} & =\epsilon M \cos \left(\mathcal{W}_{3}\right) \mathcal{W}_{6}^{2} \\
M \dot{\mathcal{W}}_{5}+\epsilon M \cos \left(\mathcal{W}_{3}\right) \dot{\mathcal{W}}_{6} & =\epsilon M \sin \left(\mathcal{W}_{3}\right) \mathcal{W}_{6}^{2} \\
-\epsilon M \sin \left(\mathcal{W}_{3}\right) \dot{\mathcal{W}}_{4}+\epsilon M \cos \left(\mathcal{W}_{3}\right) \dot{\mathcal{W}}_{5}+\left(I_{d}+\epsilon^{2} M\right) & -c_{y} \mathcal{W}_{6}=\frac{N \Phi^{2}}{2 R}\left(\Omega-\mathcal{W}_{2}+\mathcal{W}_{6}\right)-c_{\theta} \mathcal{W}_{6}+\mathcal{C}_{\theta}
\end{aligned}
$$

This can then be solved using MATLAB's built in ODE solver ode45, [26].

\section{B.2 Numerical solution for a flexible rotor shaft on rigid supports}

The equations of motion (6.6) become

$$
\begin{aligned}
\dot{\mathcal{W}}_{1} & =\mathcal{W}_{4}, \\
\dot{\mathcal{W}}_{2} & =\mathcal{W}_{5}, \\
\dot{\mathcal{W}}_{3} & =\mathcal{W}_{6}, \\
M \dot{\mathcal{W}}_{4}-\epsilon M \sin \left(\mathcal{W}_{3}\right) \dot{\mathcal{W}}_{6} & =\epsilon M \cos \left(\mathcal{W}_{3}\right) \mathcal{W}_{6}^{2}-c_{x} \mathcal{W}_{4}-k_{x} \mathcal{W}_{1}+\mathcal{C}_{x}, \\
M \dot{\mathcal{W}}_{5}+\epsilon M \cos \left(\mathcal{W}_{3}\right) \dot{\mathcal{W}}_{6} & =\epsilon M \sin \left(\mathcal{W}_{3}\right) \mathcal{W}_{6}^{2}-c_{y} \mathcal{W}_{5}-k_{y} \mathcal{W}_{2}+\mathcal{C}_{y}
\end{aligned}
$$

$$
\begin{aligned}
\left(M \mathcal{W}_{2}-\epsilon M \sin \left(\mathcal{W}_{3}\right)\right) \dot{\mathcal{W}}_{4}+ & \left(\epsilon M \cos \left(\mathcal{W}_{3}\right)-M \mathcal{W}_{1}\right) \dot{\mathcal{W}}_{5} \\
+\left(I_{d}+\epsilon^{2} M-\right. & \left.\epsilon M \mathcal{W}_{1} \cos \left(\mathcal{W}_{3}\right)-\epsilon M \mathcal{W}_{2} \sin \left(\mathcal{W}_{3}\right)\right) \dot{\mathcal{W}}_{6}= \\
& +\frac{N \Phi^{2}}{2 R}\left(\Omega-\mathcal{W}_{6}\right)-c_{\theta} \mathcal{W}_{6} \\
& -\epsilon M \mathcal{W}_{1} \mathcal{W}_{6}^{2} \sin \left(\mathcal{W}_{3}\right)+\epsilon M \mathcal{W}_{2} \mathcal{W}_{6}^{2} \cos \left(\mathcal{W}_{3}\right)+\mathcal{C}_{\theta}
\end{aligned}
$$

This can then be solved using MATLAB's built in ODE solver ode45 [26]. 